\title{
A PARTIAL FACTORIZATION OF THE POWERSUM FORMULA
}

\author{
JOHN MICHAEL NAHAY
}

Received 23 January 2004

\begin{abstract}
For any univariate polynomial $P$ whose coefficients lie in an ordinary differential field $\mathbb{E}$ of characteristic zero, and for any constant indeterminate $\alpha$, there exists a nonunique nonzero linear ordinary differential operator $k$ of finite order such that the $\alpha$ th power of each root of $P$ is a solution of $\varkappa z^{\alpha}=0$, and the coefficient functions of $\varkappa$ all lie in the differential ring generated by the coefficients of $P$ and the integers $\mathbb{Z}$. We call $\varkappa$ an $\alpha$-resolvent of $P$. The author's powersum formula yields one particular $\alpha$-resolvent. However, this formula yields extremely large polynomials in the coefficients of $P$ and their derivatives. We will use the $A$ hypergeometric linear partial differential equations of Mayr and Gelfand to find a particular factor of some terms of this $\alpha$-resolvent. We will then demonstrate this factorization on an $\alpha$-resolvent for quadratic and cubic polynomials.
\end{abstract}

2000 Mathematics Subject Classification: 12H05, 13 N15.

1. Introduction. Factoring polynomials in several variables is often a daunting, computationally intensive task. When those variables bear certain algebraic relations among each other, the factoring can become considerably easier. It is often the case that a polynomial can be too big to factor on a computer, so algebraic identities must be used to aid the factorization. In the case of computing a differential resolvent of a polynomial, one often looks for the resolvent of lowest possible order and/or weight. We call such a resolvent the Cohnian of the polynomial. The author in [9] has computed upper bounds on the weight of the Cohnian for polynomials whose distinct roots are differentially independent over constants.

In order to get to the Cohnian, one must first use the powersum formula [10] to compute a certain multiple of the Cohnian. This multiple is extremely large, resulting in an intermediate blowup problem. For example, the Cohnian of a cubic polynomial with three indeterminate coefficients fits on the $12 \mathrm{~GB}$ harddrive of a desktop computer. But it is impossible to use the powersum formula with Mathematica 4.0 on an ordinary desktop to compute a resolvent of such a cubic. This paper attempts to circumvent the intermediate blowup problem by algebraically factoring some of the terms of the resolvent with the use of partial differential equations, matrices, and a lot of linear algebra. This factoring culminates in a reduction in the weight of some of the terms of the resolvent by an amount given by (8.7) in Remark 8.3 .

DEFINITION 1.1. Let the $N$ th degree monic polynomial $P(t) \equiv \sum_{i=0}^{N}(-1)^{N-i} e_{N-i} \cdot t^{i}$ with coefficients $e \equiv\left\{e_{i}\right\}_{i=1}^{N}$ have $n$ distinct roots $\left\{z_{j}\right\}_{j=1}^{n}$. Let $\alpha$ be a transcendental constant over the ordinary differential field, $\mathbb{Q}\langle e\rangle$, generated by the rational numbers 
$\mathbb{Q}$ and the coefficients $e$ with derivation $D$. For each root $z_{j}$, let $y_{j}$ denote a nonzero solution of the logarithmic differential equation, $D y_{j} / y_{j}=\alpha \cdot\left(D z_{j} / z_{j}\right)$.

Such a solution is guaranteed to exist by [3]. We call $y_{j}$ the $\alpha$ th power of the root $z_{j}$. For convenience, we will drop the index $j$ and use the letter $z$ to denote any one of the roots $\left\{z_{j}\right\}_{j=1}^{n}$. We will use $y$ to denote any given integer power of $z$ as well as the $\alpha$ th power of $z$, depending upon the context.

By Theorem A.3 in the appendix (originally [7, Theorem 37, page 67]), there exist linear ordinary differential equations of finite order of the form $\mathfrak{z} y \equiv \sum_{m=0}^{r} \sum_{i=0}^{\Omega-m} R_{i, m}$. $\alpha^{i} D^{m} y=0$ such that each $y$ is a solution, all the coefficient functions $R_{i, m}$ (terminology of [7, page 725]) lie in $\mathbb{Q}\langle e\rangle$, and not all $R_{i, m}$ are zero. In this equation, $\Omega \equiv r \cdot(r-$ $1) / 2+1$, where the order $r$ is greater than or equal to the number of $y$ which are linearly independent over constants. We call such a linear ordinary differential equation, and any of its differential consequences, an $\alpha$ th-power ordinary differential resolvent of the polynomial $P$, or simply an $\alpha$-resolvent. By multiplying through by a common denominator, we may take all the coefficient functions $R_{i, m}$ of the resolvent $\mathfrak{z}$ to lie in $\mathbb{Z}\{e\}$, the differential ring generated by the $e$ over the ring of integers $\mathbb{Z}$. We say such a resolvent is integral. To perform the factorization in Theorem 8.2, we will need to use several nonzero linear partial differential equations which are satisfied by each $y$ and whose terms lie in $\mathbb{Q}\{e\}$. We call these linear partial differential equations $\alpha$ th-power partial differential resolvents of $P$.

DEFINITION 1.2. For any particular $\alpha$-resolvent $\sum_{(i, m)} R_{i, m} \cdot \alpha^{i} D^{m} y=0$, denote by $S$ the set of pairs $(i, m)$ such that $R_{i, m} \neq 0$.

Under the stringent condition that the coefficient ring $\mathbb{Z}\{e\}$ is a unique factorization domain, such as the case under study in this paper when all the roots of $P$ are differentially independent over constants, the weight of monomials in $\mathbb{Z}\{e\}$ is given by Definition 3.4. We can then define the Cohnian of a polynomial as the particular $\alpha$ resolvent of $P$ which is minimal first in order, then minimal in the weight of its terms. (It is conjectured that one gets the same resolvent by minimizing by weight first and then by order.) Specifically, there exists no common factor among the terms of the Cohnian in the differential ring $\mathbb{Z}\{e\}$ except \pm 1 . In the case when all the roots of $P$ are differentially independent over constants, $r=N=n, \Omega=n \cdot(n-1) / 2+1$, and we will denote the Cohnian by $\sum_{m=0}^{n} \sum_{i=0}^{\Omega-m} \theta_{i, m} \cdot \alpha^{i} D^{m} y=0$, where $\operatorname{gcd}_{(i, m) \in S}\left(\theta_{i, m}\right)=1$. By $[6$, Theorem 40, page 71] $S$ equals the complete set of pairs $(i, m)$ indicated in this double summation, excluding $(0,0)$. We will define $\Omega$ in Theorem A.3 for the more general case of resolvents for polynomials whose roots' $\alpha$ th powers bear linear relations over constants. But until the appendix, we give

$$
\Omega=\frac{n \cdot(n-1)}{2}+1
$$

as the formula for $\Omega$, consistent with the definition given in Theorem A.3 in the case of the Cohnian of a polynomial whose roots are differentially independent over constants.

If the distinct roots of $P$ are differentially independent over constants, the author's powersum formula [10] yields a nonzero multiple $\sum_{m=0}^{n} \sum_{i=0}^{\Omega-m} F_{i, m} \cdot \alpha^{i} D^{m} y=0$ of 
the Cohnian [7, Theorem 4.1] that has a weight many times larger than the weight of the Cohnian. Under the more stringent condition that all the roots of $P$ are differentially independent over constants, Theorem 8.2, the main factorization theorem, assures us that the determinant of a certain matrix, later called $2 \mathfrak{n}$, whose entries lie in $\mathbb{Q}\{e\}$, divides certain terms of the resolvent, specifically $F_{\Omega-m, m}, F_{0, m}$, and $F_{1,0}$. We will apply Theorem 8.2 to a quadratic polynomial, in which all the terms happen to factor. We conjecture that det2h divides all the coefficient functions $F_{i, m}$ in $\mathbb{Q}\{e\}$. If so, we seek formulae for the quotient $\vartheta_{i, m}$ from which we hope to extract yet another unknown factor $\Xi$ to arrive at the Cohnian $\theta_{i, m}$. Thus, we wish to complete the factorization

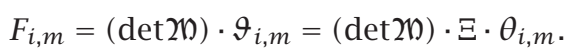

The author has also applied Theorem 8.2 in [6, Example 77, page 135] to a cubic polynomial whose coefficients are differentially independent over constants. Theorem 8.2 guarantees that eight of the thirteen coefficient functions of the Cohnian of the cubic possess the same factor. Massive computations with the computer algebra system Mathematica verified the conjecture that the determinant of $2 \mathrm{~b}$ divides all thirteen coefficient functions of the Cohnian. Unfortunately, the author was unsuccessful in reducing the size of these programs for this particular case of the cubic for this paper. It is for this reason that much algebraic work must still be done to factor resolvents before implementing them on a computer algebra system. However, the author successfully tested the conjecture on the particular cubic

$$
z^{3}-x \cdot z^{2}+x^{2} \cdot z-\left(3 x^{3}+2 x^{2}-11 x-10\right)=0
$$

to be shown in Section 12.

The very first differential resolvent had been discovered by Sir James Cockle in 1860. Writing on Cockle's work, the Reverend Robert Harley coined the term differential resolvent in 1862 [2]. Both authors considered only polynomials in the ring $\mathbb{Q}(x)[t]$. Since then, the present author has related the first-power resolvent of a quadratic polynomial to the nonlinear Riccati differential equation [8].

2. Weights, lengths, and multi-index notation for partial derivatives. Let $\mathbb{N}$ denote the set of positive integers. Let $\mathbb{N}_{0}$ denote the set of nonnegative integers. For any $n \in \mathbb{N}$, let $\mathbb{N}_{0}^{n}$ denote the set of ordered $n$-tuples of nonnegative integers.

Definition 2.1. For any $m \in \mathbb{N}$, define $[m] \equiv\{k \in \mathbb{N} \ni 1 \leq k \leq m\}$.

Definition 2.2. For any $m \in \mathbb{N}_{0}$, define $[m]_{0} \equiv\left\{k \in \mathbb{N}_{0} \ni 0 \leq k \leq m\right\}$.

DEFINITION 2.3. For any integers $i$ and $j$, define $\delta_{i, j} \equiv 1$ if $i=j$ and $\delta_{i, j}=0$ if $i \neq j$.

DEFINITION 2.4. For any $m \in \mathbb{N}$, let $\mathrm{I}_{m}$ denote the $m \times m$ identity matrix.

Definition 2.5. For any matrix $\mathrm{M}$, let $\mathrm{M}^{\dagger}$ denote its transpose, and let $|\mathrm{M}|$ or $\operatorname{det} \mathrm{M}$ denote its determinant. All rings and fields will be assumed to have characteristic zero.

Definition 2.6. For any finite set $X$, let $|X|$ denote the size of $X$. 
Let $n \in \mathbb{N}$. On the ordinary differential field $\mathbb{Q}\langle e\rangle$ apply the partial derivations $\left\{\partial_{1, i}\right\}_{i=1}^{n}$ such that $\partial_{1, i} e_{j}=\delta_{i, j}$. In other words, for each $i \in[n], \partial_{1, i}$ is the natural derivation with respect to $e_{i}$ holding all other $e_{j \neq i}$ fixed. For each $m \in \mathbb{N}$, denote the $m$ th composition of $\partial_{1, i}$ by $\partial_{1, i}^{m}$. For each $k \in \mathbb{N}_{0}$, define $e_{j}^{(k)} \equiv D^{k} e_{j}$, where $D^{0} e_{j} \equiv e_{j}$.

Let $\lambda_{1}, \ldots, \lambda_{n}, \imath$ be $n+1$ nonnegative integers with $\imath \geq \sum_{k=1}^{n} \lambda_{k}$. Let $\lambda$ represent the ordered $n$-tuple, $\left(\lambda_{1}, \ldots, \lambda_{n}\right) \in \mathbb{N}_{0}^{n}$. We will call $n$ the dimension of $\lambda$. The multinomial function is defined by

$$
\left(\begin{array}{c}
\imath \\
\lambda
\end{array}\right) \equiv\left(\begin{array}{c}
\imath \\
\lambda_{1}, \ldots, \lambda_{n}
\end{array}\right) \equiv \frac{\imath !}{\left(\imath-\sum_{k=1}^{n} \lambda_{k}\right) ! \cdot \prod_{k=1}^{n} \lambda_{k} !} .
$$

Definition 2.7. Define the weight of $\lambda$ to be $\sum_{k=1}^{n} k \cdot \lambda_{k}$ and denote it by $|\lambda|$.

DEFINITION 2.8. Define the length of $\lambda$ to be $\sum_{k=1}^{n} \lambda_{k}$ and denote it by $\ell(\lambda)$.

These definitions and notations are related to the definitions and notations used in [4] in the following way. Define a finite nonincreasing sequence, $v \equiv\left(v_{j}\right)$, of positive integers consisting of $\lambda_{k} k$ 's for each $k \in[n]$. Then, we say $v$ is a partition of the integer, $|\lambda|$, with the same weight and length as $\lambda$. We say $\lambda_{k}$ is the multiplicity of $k$ in $v$ and write $v=\left(1^{\lambda_{1}} 2^{\lambda_{2}} \cdots n^{\lambda_{n}}\right)$. For reference, there exist only finitely many $\lambda \in \mathbb{N}_{0}^{n}$ of any given weight or any given length. The $n$-tuple $(0)^{n}$ is the only $n$-tuple with $\ell=0$ and $w=0$.

DEFINITION 2.9. For any $\lambda \in \mathbb{N}_{0}^{n}$, define the partial derivative operator, $\partial^{\lambda}$, on $\mathbb{Q}\langle e\rangle$ to be $\partial_{1,1}^{\lambda_{1}} \partial_{1,2}^{\lambda_{2}} \cdots \partial_{1, n}^{\lambda_{n}}$. Define the weight of $\partial^{\lambda}$ to be $|\lambda|$ given by Definition 2.7. Define the length or order of $\partial^{\lambda}$ to be $\ell(\lambda)$ given by Definition 2.8 .

By a well-known theorem in [5], we have the partial differential resolvent

$$
\partial^{\lambda} y=\partial^{\sigma} y
$$

if $\ell(\lambda)=\ell(\sigma)$ and $|\lambda|=|\sigma|$. Hence, we may indicate the partial derivative operator, $\partial^{\lambda}$, simply by the length, $\ell$, and weight, $w$, of $\lambda$ and denote it by $\partial_{\ell, w}$. We will separate $\ell$ and $w$ in the subscript of $\partial_{\ell, w}$ with a comma. Hence, the derivation $\partial_{1, j}$ has length 1 and weight $j$ for each $j \in[n]$. For any $\ell \in \mathbb{N}, \partial_{\ell, w}$ exists only for $w$ in the range, $\ell \leq w \leq \ell \cdot n$. The unique partial derivative of order $\ell$ and minimal weight $w=\ell$ is $\partial_{\ell, \ell}$. The unique partial derivative of order $\ell$ and maximal weight $w=\ell \cdot n$ is $\partial_{\ell, \ell \cdot n}$.

3. Powersums and the powersum formula. When the roots of $P$ are distinct, for each $q \in \mathbb{Z}$, define the $q$ th powersum of the roots to be $p_{q} \equiv \sum_{j=1}^{n} z_{j}^{q}$. By the determinantal formula given by [4, page 28], we have that $p_{q} \in \mathbb{Z}[e]$, the nondifferential ring generated by the $e$ and $\mathbb{Z}$, for each $q \in \mathbb{N}_{0}$.

DEFINITION 3.1. For any $m \in \mathbb{N}_{0}$, define $\mathbb{Z}\{e\}_{m}$ to be the (nondifferential) ring generated by the coefficients $e$ of the polynomial $P$ of Definition 1.1, their derivatives up through $m$ th order, and the integers. Define $\mathbb{Q}\{e\}_{m}$ to be the (nondifferential) ring generated by $e$, their derivatives up through $m$ th order, and the rationals. 
A differential ring must contain infinitely many derivatives of all its members. Definition 1.1 implies $\mathbb{Z}\{e\}_{0}=\mathbb{Z}[e]$ and $D^{m} q^{i} p_{q} \in \mathbb{Z}\{e\}_{m} \subset \mathbb{Z}\{e\}$ for every $m, i, q \in \mathbb{N}_{0}$.

From Section 5 onward it is important to remember that the derivation, $D$, is a $\mathbb{Z}$-homomorphic map $D: \mathbb{Z}\{e\}_{m} \rightarrow \mathbb{Z}\{e\}_{m+1}$ which may be expressed as $D=$ $\sum_{k=0}^{m} \sum_{j=1}^{n} e_{j}^{(k+1)}\left(\partial / \partial e_{j}^{(k)}\right)$ on the ring, $\mathbb{Z}\{e\}_{m}$, where we have extended the natural derivation, $\partial_{1, i} e_{j}=\delta_{i, j}$, to $\left(\partial / \partial e_{j}^{(k)}\right) e_{i}^{(l)} \equiv \delta_{i, j} \cdot \delta_{k, l}$. It is worth mentioning that $\mathbb{Z}\{e\}_{m}$ is a partial differential ring with derivations, $\partial / \partial e_{j}^{(k)}$, with $k \in[m]_{0}$ and $j \in[n]$, but does not possess $D$ as a derivation.

While partial differential resolvent (2.2) implies that $\partial^{\lambda} y_{1}=\partial^{\sigma} y_{1}$ and $\partial^{\lambda} y_{2}=\partial^{\sigma} y_{2}$, when $\ell(\lambda)=\ell(\sigma)$ and $|\lambda|=|\sigma|$ it is important to note that $\partial^{\lambda}\left(y_{1} y_{2}\right) \neq \partial^{\sigma}\left(y_{1} y_{2}\right)$. Hence, although it follows that $\partial^{\lambda} p_{q}=\partial^{\sigma} p_{q}$ for all $q \in \mathbb{Z}$ by summing $\partial^{\lambda} y=\partial^{\sigma} y$ over all the roots of $P$, it does not follow that $\partial^{\lambda}\left(p_{q} \cdot p_{r}\right)=\partial^{\sigma}\left(p_{q} \cdot p_{r}\right)$.

For example, for the cubic $z^{3}-e_{1} \cdot z^{2}+e_{2} \cdot z-e_{3}=0$, one easily sees that $\lambda=(1,2,0)$ and $\sigma=(2,0,1)$ have equal length, $\ell(\lambda)=\ell(\sigma)=3$, and equal weight, $|\lambda|=1 \cdot 1+2$. $2+3 \cdot 0=5,|\sigma|=1 \cdot 2+2 \cdot 0+3 \cdot 1=5$. So $\partial^{\lambda} y=\partial_{1,1}^{1} \partial_{1,2}^{2} y=\partial_{1,1}^{2} \partial_{1,3}^{1} y=\partial^{\sigma} y$ is correct, where $y=z^{q}$ for any $q \in \mathbb{Z}$. But $\partial^{\lambda}\left(e_{1} e_{2}^{2}\right)=\partial_{1,1}^{1} \partial_{1,2}^{2}\left(e_{1} e_{2}^{2}\right)=2 \neq 0=\partial_{1,1}^{2} \partial_{1,3}^{1}\left(e_{1} e_{2}^{2}\right)=$ $\partial^{\sigma}\left(e_{1} e_{2}^{2}\right)$. Henceforth, when we write $\partial^{\lambda}=\partial^{\sigma}$, we imply that this partial differential resolvent and all other partial differential resolvents of $P$ hold only when the operators are applied to $y$ or the powersums $p_{q}$. We will omit the $y$ or $p_{q}$ for convenience when necessary.

DEFINITION 3.2. Define $\Phi$ to be the number of nonzero coefficient-functions $R_{i, m}$ in a resolvent $\mathfrak{Z}=\sum_{(i, m)} R_{i, m} \cdot \alpha^{i} \cdot D^{m} y$.

So $\Phi$ equals the size of the set $S$ given by Definition 1.2.

DEFINITION 3.3. Define $\Psi \equiv \Phi-1$.

In general, one does not know a priori the value of $\Phi$ for a given polynomial until one starts computing $\mathfrak{Z}$. The powersum formula [10, Corollary 4.3] offers one possible way to compute the $R_{i, m}$. To derive the formula, one first orders the pairs $(i, m)$ in $S$. Let $\operatorname{sgn}(i, m)$ denote the position of the pair $(i, m)$ in this ordering. Then, one sets $R_{i, m}=F_{i, m}$ as in [10] to get the powersum formula

$$
A_{i, m} \equiv\left[D^{m^{\prime}} q^{i^{\prime}} p_{q}\right]_{\substack{\left(i^{\prime}, m^{\prime}\right) \neq(i, m) \\\left(i^{\prime}\right) \times q}}, \quad F_{i, m} \equiv(-1)^{\operatorname{sgn}(i, m)} \cdot \operatorname{det} A_{i, m}
$$

Here, $A_{i, m}$ is the $\Psi \times \Psi$ matrix with rows labelled by $\left(i^{\prime}, m^{\prime}\right)$ and columns labelled by $q$, where we take $q$ to lie in the smallest possible set of $\Psi$ nonnegative integers, which is $[\Psi]$. This is basically an application of Cramer's rule.

Since $q^{i} D^{m} p_{q} \in \mathbb{Z}\{e\}$ for every $q \in \mathbb{N}_{0}$, we could choose $q$ not to lie in [ $\Psi$ ] but to lie in $\Gamma$ for any subset $\Gamma \subset \mathbb{N}_{0}$ of $\Psi$ distinct nonnegative integers. However, in the case that the $e$ are differentially independent over $\mathbb{Z}$, the weight of monomials in $\mathbb{Z}\{e\}$ is well defined.

DEFINITION 3.4. Define the weight of the monomial $s \cdot \prod_{j, k}\left(e_{j}^{(k)}\right)^{\pi_{j, k}}$ with $s \in \mathbb{Z}$, $s \neq 0$, and $\pi_{j, k} \in \mathbb{N}_{0}$ to be $\sum_{j, k} j \cdot \pi_{j, k}$. 
By Definition 3.4 the weight of integers is zero. The weight of $p_{q}$ is $q$. Therefore the powersum formula has been defined for any polynomial such that, in the case of differentially independent roots, the formula yields a resolvent with terms in $\mathbb{Z}\{e\}$ of minimal weight over all other possible choices of positive integers $q$. This will minimize the amount of factoring necessary to get to the Cohnian. Since the polynomial $\operatorname{det}\left[q^{i} D^{m} p_{q}\right]_{(i, m) \times q}$ is homogeneous of weight $\sum_{q \in \Gamma} q$, this weight is minimized by choosing $\Gamma=[\Psi]$ and equals $\Psi \cdot(\Psi+1) / 2$.

4. Examples of low-order resolvents. By Theorem A.3 in the appendix, polynomials whose $n$ distinct roots have $\alpha$ th powers which are linearly independent over constants possess differential resolvents of the following forms. By [6, Theorem 40, page 71] or [7, Theorem 4.1], and in the smaller cases by direct computation, it is known that all $F_{i, m} \neq 0$ in each of these resolvents.

Two DisTINCT ROOTS. $n=2, \Omega=2, \Phi=5 . P(t)=(t-u)^{\pi_{u}} \cdot(t-v)^{\pi_{v}}$ has a resolvent of the form

$$
F_{0,2} \cdot D^{2} y+\left(F_{0,1}+F_{1,1} \cdot \alpha\right) \cdot D y+\left(F_{1,0} \cdot \alpha+F_{2,0} \cdot \alpha^{2}\right) \cdot y=0
$$

The set $S$ given by Definition 1.2 for resolvent (4.1) is

$$
\{(0,2),(0,1),(1,1),(1,0),(2,0)\}
$$

By [8, Theorem 1], the logarithmic derivative $D z / z$ satisfies the first-order inhomogeneous linear ordinary differential equation

$$
F_{0,2} \cdot D\left(\frac{D z}{z}\right)+F_{0,1} \cdot\left(\frac{D z}{z}\right)+F_{1,0}=0
$$

and the quadratic polynomial

$$
F_{0,2} \cdot\left(\frac{D z}{z}\right)^{2}+F_{1,1} \cdot\left(\frac{D z}{z}\right)+F_{2,0}=0
$$

THREE DISTINCT ROOTS. $n=3, \Omega=4, \Phi=13 . P(t)=(t-u)^{\pi_{u}}(t-v)^{\pi_{v}}(t-w)^{\pi_{w}}$ has a resolvent of the form

$$
\begin{aligned}
\left(F_{0,3}\right. & \left.+F_{1,3} \cdot \alpha\right) \cdot D^{3} y+\left(F_{0,2}+F_{1,2} \cdot \alpha+F_{2,2} \cdot \alpha^{2}\right) \cdot D^{2} y \\
& +\left(F_{0,1}+F_{1,1} \cdot \alpha+F_{2,1} \cdot \alpha^{2}+F_{3,1} \cdot \alpha^{3}\right) \cdot D y \\
& +\left(F_{1,0} \cdot \alpha+F_{2,0} \cdot \alpha^{2}+F_{3,0} \cdot \alpha^{3}+F_{4,0} \cdot \alpha^{4}\right) \cdot y=0 .
\end{aligned}
$$

The set $S$ given by Definition 1.2 for resolvent (4.5) is

$$
\{(0,3),(1,3),(0,2),(1,2),(2,2),(0,1),(1,1),(2,1),(3,1),(1,0),(2,0),(3,0),(4,0)\} \text {. }
$$


By [8, Theorem 1], the logarithmic derivative $D z / z$ satisfies the second-order inhomogeneous linear ordinary differential equation

$$
F_{0,3} \cdot D^{2}\left(\frac{D z}{z}\right)+F_{0,2} \cdot D\left(\frac{D z}{z}\right)+F_{0,1} \cdot\left(\frac{D z}{z}\right)+F_{1,0}=0
$$

and the cubic polynomial

$$
F_{1,3} \cdot\left(\frac{D z}{z}\right)^{3}+F_{2,2} \cdot\left(\frac{D z}{z}\right)^{2}+F_{3,1} \cdot\left(\frac{D z}{z}\right)+F_{4,0}=0
$$

FOUR DISTINCT ROOTS. $n=4, \Omega=7, \Phi=29 . P(t)=(t-u)^{\pi_{u}}(t-v)^{\pi_{v}}(t-w)^{\pi_{w}}(t-$ $x)^{\pi_{x}}$ has a resolvent of the form

$$
\begin{aligned}
\left(F_{0,4}\right. & \left.+F_{1,4} \cdot \alpha+F_{2,4} \cdot \alpha^{2}+F_{3,4} \cdot \alpha^{3}\right) \cdot D^{4} y \\
& +\left(F_{0,3}+F_{1,3} \cdot \alpha+F_{2,3} \cdot \alpha^{2}+F_{3,3} \cdot \alpha^{3}+F_{4,3} \cdot \alpha^{4}\right) \cdot D^{3} y \\
& +\left(F_{0,2}+F_{1,2} \cdot \alpha+F_{2,2} \cdot \alpha^{2}+F_{3,2} \cdot \alpha^{3}+F_{4,2} \cdot \alpha^{4}+F_{5,2} \cdot \alpha^{5}\right) \cdot D^{2} y \\
& +\left(F_{0,1}+F_{1,1} \cdot \alpha+F_{2,1} \cdot \alpha^{2}+F_{3,1} \cdot \alpha^{3}+F_{4,1} \cdot \alpha^{4}+F_{5,1} \cdot \alpha^{5}+F_{6,1} \cdot \alpha^{6}\right) \cdot D y \\
& +\left(F_{1,0} \cdot \alpha+F_{2,0} \cdot \alpha^{2}+F_{3,0} \cdot \alpha^{3}+F_{4,0} \cdot \alpha^{4}+F_{5,0} \cdot \alpha^{5}+F_{6,0} \cdot \alpha^{6}+F_{7,0} \cdot \alpha^{7}\right) \cdot y=0 .
\end{aligned}
$$

By [8, Theorem 1], the logarithmic derivative $D z / z$ satisfies the third-order inhomogeneous linear ordinary differential equation

$$
F_{0,4} \cdot D^{3}\left(\frac{D z}{z}\right)+F_{0,3} \cdot D^{2}\left(\frac{D z}{z}\right)+F_{0,2} \cdot D\left(\frac{D z}{z}\right)+F_{0,1} \cdot\left(\frac{D z}{z}\right)+F_{1,0}=0
$$

and the quartic polynomial

$$
F_{3,4} \cdot\left(\frac{D z}{z}\right)^{4}+F_{4,3} \cdot\left(\frac{D z}{z}\right)^{3}+F_{5,2} \cdot\left(\frac{D z}{z}\right)^{2}+F_{6,1} \cdot\left(\frac{D z}{z}\right)+F_{7,0}=0 .
$$

Basically, [8, Theorem 1] says that the logarithmic derivatives of the roots satisfy an inhomogeneous $(n-1)$ th-order linear ordinary differential equation whose terms $F_{0, m}$ and $F_{1,0}$ are the coefficients of the lowest degree in $\alpha$ in the $\alpha$-resolvent of $P$. By a slight extension of the definition of a differential resolvent to include inhomogeneous linear ordinary differential equations, we may say that the powersum formula (3.1) also yields a 1-resolvent for the logarithmic derivatives of the roots of $P$. Also [8, Theorem 1] says that the logarithmic derivatives of the roots satisfy the $n$ th-degree polynomial whose coefficients are the terms $F_{\Omega-m, m}$, which are the coefficients of the highest degree in $\alpha$ in the $\alpha$-resolvent of $P$. Therefore, [8, Theorem 1] provides a way of partially factoring all the terms of a minimal-order inhomogeneous 1-resolvent and of finding the minimal polynomial of the logarithmic derivatives of the roots of $P$ over $\mathbb{Q}\{e\}$.

\section{Partial differential resolvents}

DEFINITION 5.1. Define the partial derivation operator $\Theta$ on the partial differential field $\mathbb{Q}\langle e\rangle$ to be $\Theta \equiv \sum_{k=1}^{n} k \cdot e_{k} \cdot \partial_{1, k}$. 
Then we have the Euler-homogeneity partial differential resolvent

$$
\Theta y=\alpha \cdot y \quad \text { or } \quad \Theta p_{q}=q \cdot p_{q}
$$

In the next theorem, we use the $A$-hypergeometric partial differential resolvents of Mayr [5] and Gelfand et al. [1] and their differential consequences to demonstrate that all compositions of the derivations $D$ and $\Theta$ can be expressed as linear combinations over $\mathbb{Q}\{e\}$ of a certain set, $\partial_{\ell, w}$, of partial derivatives of distinct weights. Although explicit formulae have been determined, for instance in [6, Theorem 59, page 109], for the expression of the operator $D^{m} \Theta^{i}$ as a linear combination of the partial derivatives, $\partial_{\ell, w}$, the computation of these formulae is not enlightening and involves no original ideas. So, these formulae have been omitted. Furthermore, on simple examples, it is easier to work out the formulae for $D^{m} \Theta^{i}$ by hand, as we will do in Section 9 for a quadratic polynomial. On more complicated examples, such as for the cubic polynomial in Section 12, it is easier to use computer algebra systems, such as Mathematica, to compute $D^{m} \Theta^{i}$. Rather than programming the explicit formulae, which involve multiple summations over many $n$-tuples, it is easier to use the partial and total differentiation capabilities of the computer algebra system to recursively compute as many $D^{m} \Theta^{i}$ as needed.

By induction on $i$ and $m$, it is possible for the reader to determine that there exist $\psi_{i, m, \lambda} \in \mathbb{Z}\{e\}_{m}$ such that $D^{m} \Theta^{i}=\sum_{\lambda} \psi_{v, m, \lambda} \cdot \partial^{\lambda}$ with the sum over all distinct $\lambda \in \mathbb{N}_{0}^{n}$. It is easy to see that $\psi_{i, m, \lambda} \neq 0$ for only finitely many $\lambda$. Specifically $\psi_{i, m, \lambda}=0$ for all $\lambda \ni \ell(\lambda)>i+m$. In Theorem 5.2 we will collect terms in this summation and express $D^{m} \Theta^{i}$ as a linear combination over $\mathbb{Q}\{e\}$ of partial derivatives $\partial^{\lambda}$ over $\lambda \in \mathbb{N}_{0}^{n}$ of distinct length and weight.

Theorem 5.2 will use the particular system [6, Theorem 49, page 100] of partial differential resolvents of $P$,

$$
\partial_{1, j} y=-\sum_{i=0}^{n} e_{i} \cdot \partial_{2, i+j} y \quad \text { for each } j \in[n], j>1 .
$$

For lack of a published reference for partial differential resolvent (5.2), the author credits the referees at the Journal of Symbolic Computation for pointing out that these partial differential resolvents are simply the statement that the partial derivative, $\partial_{1, j} y$, of a function with standard homogeneity 0 has homogeneity -1 . By repeated differentiation of partial differential resolvent (5.2), one may determine as in [6, Theorem 56, page 105] the following partial differential resolvents of $P$ for any $\ell, w, \varpi \in \mathbb{N}$ with $\varpi \geq w>\ell$ of the form

$$
\partial_{\ell, w}=\sum_{\kappa=w}^{\kappa=\varpi} f_{\kappa} \cdot \partial_{\kappa, \kappa}+\sum_{\kappa=\varpi+1}^{\kappa=n \cdot(\varpi-\ell)+w} f_{\kappa} \cdot \partial_{\varpi, \kappa},
$$

where $f_{\sigma} \in \mathbb{Q}[e]$ depend upon $\varpi, w$, and $\ell$. We will use partial differential resolvent (5.3) later to perform a simple computation for a quadratic polynomial. Partial differential resolvent (5.3) expresses a partial derivative of a given length and weight as a linear combination of partial derivatives of higher lengths and weights. 
THEOREM 5.2. Let $i, m, \varpi \in \mathbb{N}_{0}$ and $\varpi \geq i+m>0$. Then

$$
D^{m} \Theta^{i}=\sum_{\kappa=1}^{\varpi} \boldsymbol{\tau}_{(i, m) \times \kappa} \cdot \partial_{\kappa, \kappa}+\sum_{\kappa=\varpi+1}^{n \cdot \varpi} \boldsymbol{\tau}_{(i, m) \times \kappa} \cdot \partial_{\varpi, \kappa} \text { for some } \boldsymbol{\tau}_{(i, m) \times \kappa} \in \mathbb{Q}\{e\} .
$$

Proof. By partial differential resolvent (2.2), we may write $\partial^{\lambda}=\partial_{\ell, w}$, where $w=$ $|\lambda|$ and $\ell=\ell(\lambda)$ are given by Definitions 2.7 and 2.8, respectively. In the summation $D^{m} \Theta^{i}=\sum_{\lambda} \psi_{i, m, \lambda} \cdot \partial^{\lambda}$ with $\psi_{i, m, \lambda} \in \mathbb{Z}\{e\}_{m}$ we collect together those $n$-tuples $\lambda \in \mathbb{N}_{0}^{n}$ with a given weight $w=|\lambda|$ and a given length $\ell=\ell(\lambda)$. Define $\mho_{i, m, \ell, w} \equiv$ $\sum_{\substack{\lambda \ni(\lambda)=\ell \\|\lambda|=w}} \psi_{i, m, \lambda} \in \mathbb{Z}\{e\}_{m}$. Then $D^{m} \Theta^{i}=\sum_{w=1}^{n \cdot(i+m)} \sum_{\ell=1}^{\min (i+m, w)} \mho_{i, m, \ell, w} \cdot \partial_{\ell, w}$.

We first separate out in this sum those partials whose length equals their weight. We then express all partials, $\partial_{\ell, w}$, with $\ell>w$ in terms of partials with lengths and weights in the range $\{(1,1),(2,2), \ldots,(\varpi, \varpi),(\varpi, \varpi+1), \ldots,(\varpi, n \cdot \varpi)\}$ using partial differential resolvent (5.3). Thus $D^{m} \Theta^{i}=\sum_{\kappa=1}^{\varpi}\left(\sum_{\ell=1}^{\kappa} \mho_{i, m, \ell, \kappa}\right) \cdot f_{\kappa} \cdot \partial_{\kappa, \kappa}+\sum_{\ell, w} \mho_{i, m, \ell, w} \sum_{\kappa=\varpi+\ell}^{n \cdot(\varpi-\ell)+w} f_{\kappa}$. $\partial_{\varpi, k}$, where the natural condition $w \leq n \cdot \ell$ guarantees that $\kappa \leq n \cdot \varpi$. Define $\tau_{(i, m) \times \kappa} \equiv$ $f_{\kappa} \cdot \sum_{\ell=1}^{\kappa} \mho_{i, m, \ell, \kappa} \in \mathbb{Q}\{e\}$ for $1 \leq \kappa \leq \varpi$ and $\tau_{(i, m) \times \kappa} \equiv f_{\kappa} \cdot \sum_{w=1}^{\kappa-n \cdot(\varpi-\ell)} \sum_{\ell=1}^{\min (w, i+m)} \mho_{i, m, \ell, w}$ $\in \mathbb{Q}\{e\}$ for $\varpi+1 \leq \kappa \leq n \cdot \varpi$. Then $D^{m} \Theta^{i}=\sum_{\kappa=1}^{\varpi} \boldsymbol{T}_{(i, m) \times \kappa} \cdot \partial_{\kappa, \kappa}+\sum_{\kappa=\varpi+1}^{n \cdot \varpi} \boldsymbol{T}_{(i, m) \times \kappa} \cdot \partial_{\varpi, \kappa}$.

DEFINITION 5.3. For particular $i, m$, and $\varpi$, a particular set $\mathcal{A}$ of partial derivatives $\partial_{\ell, w}$ represents the operator $D^{m} \Theta^{i}$ if $D^{m} \Theta^{i}$ can be expressed as a linear combination over the differential ring $\mathbb{Q}\{e\}$ of the partials from $\mathcal{A}$ as in Theorem 5.2.

It is important to remember that the transition coefficients $\tau_{(i, m) \times k}$ appearing in Theorem 5.2 depend upon the choice of $\varpi$. In Theorem 7.1 , when we choose $\varpi=\Omega$, we will use $\tau_{(i, m) \times \kappa}$, and in Theorem 8.1 , when we choose $\varpi=L$, we will use $\hat{\tau}_{(i, m) \times \kappa}$.

\section{Demonstration of Theorem 5.2}

EXAMPLE 6.1. Let $P(t)=t^{3}-e_{1} \cdot t^{2}+e_{2} \cdot t-e_{3}$ be a cubic with three distinct roots. Express $D^{2} y$ as a sum of terms in $\mathbb{Q}\{e\}$ times partial derivatives of $y$ with respect to $e_{1}, e_{2}$, and $e_{3}$ of order up to $\varpi=3$ and with distinct weight up to $n \cdot \varpi=9$.

Solution. We add no complications by considering the expansion of $y=z^{\alpha}$ for any indeterminate power $\alpha$ of any root $z$ of $P$ until the final computation to check that both sides of the identity in Theorem 5.2 are equal. It is practically impossible to write a formula for $z^{\alpha}$ as a function of $e_{1}, e_{2}$, and $e_{3}$ and then to explicitly compute the partial derivatives of $z^{\alpha}$ with respect to $e_{1}, e_{2}$, and $e_{3}$. Instead, we test only the final partial differential resolvent with a computer algebra system by applying the final partial differential resolvent to at least nine powersums.

First apply

$$
D=e_{1}^{\prime} \frac{\partial}{\partial e_{1}}+e_{2}^{\prime} \frac{\partial}{\partial e_{2}}+e_{3}^{\prime} \frac{\partial}{\partial e_{3}}
$$

to $y$. Now apply $D$ again, where this second derivative is given by

$$
D=e_{1}^{\prime} \frac{\partial}{\partial e_{1}}+e_{2}^{\prime} \frac{\partial}{\partial e_{2}}+e_{3}^{\prime} \frac{\partial}{\partial e_{3}}+e_{1}^{\prime \prime} \frac{\partial}{\partial e_{1}^{\prime}}+e_{2}^{\prime \prime} \frac{\partial}{\partial e_{2}^{\prime}}+e_{3}^{\prime \prime} \frac{\partial}{\partial e_{3}^{\prime}}
$$


since $e_{1}^{\prime}, e_{2}^{\prime}$, and $e_{3}^{\prime}$ appear in $D y$. By partial differential resolvent (2.2) we may equate partial derivatives of equal order and weight. In this case, $\partial^{2} y / \partial e_{1} \partial e_{3}=\partial^{2} y / \partial e_{2}^{2}$. For a quadratic, there are no distinct partial derivatives of equal length (order) and weight, since

$$
\begin{aligned}
& \frac{\partial u+v}{\partial e_{1}^{u} \partial e_{2}^{v}}=\frac{\partial u^{\prime}+v^{\prime}}{\partial e_{1}^{u^{\prime}} \partial e_{2}^{v^{\prime}}} \\
& \Longleftrightarrow\left\{u+v=u^{\prime}+v^{\prime}, 1 \cdot u+2 \cdot v=1 \cdot u^{\prime}+2 \cdot v^{\prime}\right\} \\
& \Longleftrightarrow\left\{u=u^{\prime}, v=v^{\prime}\right\} .
\end{aligned}
$$

Thus, we chose a cubic to demonstrate distinct partials of equal length and weight. We get

$$
\begin{aligned}
D^{2} y= & \left(e_{1}^{\prime}\right)^{2} \frac{\partial^{2} y}{\partial e_{1}^{2}}+2 e_{1}^{\prime} e_{2}^{\prime} \frac{\partial^{2} y}{\partial e_{1} \partial e_{2}}+\left(2 e_{1}^{\prime} e_{3}^{\prime}+\left(e_{2}^{\prime}\right)^{2}\right) \frac{\partial^{2} y}{\partial e_{1} \partial e_{3}} \\
& +2 e_{2}^{\prime} e_{3}^{\prime} \frac{\partial^{2} y}{\partial e_{2} \partial e_{3}}+\left(e_{3}^{\prime}\right)^{2} \frac{\partial^{2} y}{\partial e_{3}^{2}}+e_{1}^{\prime \prime} \frac{\partial y}{\partial e_{1}}+e_{2}^{\prime \prime} \frac{\partial y}{\partial e_{2}}+e_{3}^{\prime \prime} \frac{\partial y}{\partial e_{3}},
\end{aligned}
$$

where we have ordered second-order and then first-order partial derivatives by increasing weight. In the notation of Section 5 , the coefficient of the partial derivative $\partial^{\lambda_{1}+\lambda_{2}+\lambda_{3}} y / \partial e_{1}^{\lambda_{1}} \partial e_{2}^{\lambda_{2}} \partial e_{3}^{\lambda_{3}}$ is $\psi_{0,2, \lambda}$.

Example 6.1 requests that we expand these partial derivatives of $y$ as sums over the ring $\mathbb{Q}\left\{e_{1}, e_{2}, e_{3}\right\}$ of partial derivatives of $y$ of order up through $\varpi=3$. More specifically, we wish to expand these partial derivatives of $y$ as sums over the ring $\mathbb{Q}\left\{e_{1}, e_{2}, e_{3}\right\}$ of the $n \cdot \varpi=9$ partial derivatives

$$
\begin{aligned}
\left\{\partial_{1,1}, \ldots, \partial_{\varpi, \varpi}, \partial_{\varpi, \varpi+1}, \ldots, \partial_{\varpi, n \cdot \varpi}\right\} & =\left\{\partial_{1,1}, \partial_{2,2}, \partial_{3,3}, \partial_{3,4}, \partial_{3,5}, \partial_{3,6}, \partial_{3,7}, \partial_{3,8}, \partial_{3,9}\right\} \\
= & \left\{\frac{\partial y}{\partial e_{1}}, \frac{\partial^{2} y}{\partial e_{1}^{2}}, \frac{\partial^{3} y}{\partial e_{1}^{3}}, \frac{\partial^{3} y}{\partial e_{1}^{2} \partial e_{2}}, \frac{\partial^{3} y}{\partial e_{1}^{2} \partial e_{3}}=\frac{\partial^{3} y}{\partial e_{1} \partial e_{2}^{2}},\right. \\
& \left.\frac{\partial^{3} y}{\partial e_{1} \partial e_{2} \partial e_{3}}=\frac{\partial^{3} y}{\partial e_{2}^{3}}, \frac{\partial^{3} y}{\partial e_{1} \partial e_{3}^{2}}=\frac{\partial^{3} y}{\partial e_{2}^{2} \partial e_{3}}, \frac{\partial^{3} y}{\partial e_{2} \partial e_{3}^{2}}, \frac{\partial^{3} y}{\partial e_{3}^{3}}\right\} .
\end{aligned}
$$

To do this we apply partial differential resolvent (5.2) with $j \in\{2,3\}$,

$$
\partial_{1,2} y=-\sum_{i=0}^{3} e_{i} \cdot \partial_{2, i+2} y, \quad \partial_{1,3} y=-\sum_{i=0}^{3} e_{i} \cdot \partial_{2, i+3} y,
$$

and their differential consequences. In traditional notation,

$$
\begin{aligned}
& \frac{\partial y}{\partial e_{2}}=-\frac{\partial^{2} y}{\partial e_{1}^{2}}-e_{1} \cdot \frac{\partial^{2} y}{\partial e_{1} \partial e_{2}}-e_{2} \cdot \frac{\partial^{2} y}{\partial e_{2}^{2}}-e_{3} \cdot \frac{\partial^{2} y}{\partial e_{2} \partial e_{3}}, \\
& \frac{\partial y}{\partial e_{3}}=-\frac{\partial^{2} y}{\partial e_{1} \partial e_{2}}-e_{1} \cdot \frac{\partial^{2} y}{\partial e_{2}^{2}}-e_{2} \cdot \frac{\partial^{2} y}{\partial e_{2} \partial e_{3}}-e_{3} \cdot \frac{\partial^{2} y}{\partial e_{3}^{2}} .
\end{aligned}
$$

We may apply $\partial / \partial e_{1}, \partial / \partial e_{2}$, and $\partial / \partial e_{3}$ to (6.7) and (6.8) to get six higher-order differential consequences. One of the resulting six partial differential resolvents is redundant 
because $\partial^{2} y /\left(\partial e_{1} \partial e_{3}\right)=\partial^{2} y / \partial e_{2}^{2}$ and another is redundant because $\partial^{2} y /\left(\partial e_{2} \partial e_{3}\right)=$ $\partial^{2} y /\left(\partial e_{3} \partial e_{2}\right)$. Thus we get four distinct partial differential resolvents

$$
\begin{aligned}
\frac{\partial^{2} y}{\partial e_{1} \partial e_{2}} & =-\frac{\partial^{3} y}{\partial e_{1}^{3}}-\frac{\partial^{2} y}{\partial e_{1} \partial e_{2}}-e_{1} \cdot \frac{\partial^{3} y}{\partial e_{1}^{2} \partial e_{2}}-e_{2} \cdot \frac{\partial^{3} y}{\partial e_{1} \partial e_{2}^{2}}-e_{3} \cdot \frac{\partial^{3} y}{\partial e_{1} \partial e_{2} \partial e_{3}}, \\
\frac{\partial^{2} y}{\partial e_{2}^{2}} & =-\frac{\partial^{3} y}{\partial e_{1}^{2} \partial e_{2}}-e_{1} \cdot \frac{\partial^{3} y}{\partial e_{1} \partial e_{2}^{2}}-\frac{\partial^{2} y}{\partial e_{2}^{2}}-e_{2} \cdot \frac{\partial^{3} y}{\partial e_{2}^{3}}-e_{3} \cdot \frac{\partial^{3} y}{\partial e_{2}^{2} \partial e_{3}}, \\
\frac{\partial^{2} y}{\partial e_{2} \partial e_{3}} & =-\frac{\partial^{3} y}{\partial e_{1}^{2} \partial e_{3}}-e_{1} \cdot \frac{\partial^{3} y}{\partial e_{1} \partial e_{2} \partial e_{3}}-e_{2} \cdot \frac{\partial^{3} y}{\partial e_{2}^{2} \partial e_{3}}-\frac{\partial^{2} y}{\partial e_{2} \partial e_{3}}-e_{3} \cdot \frac{\partial^{3} y}{\partial e_{2} \partial e_{3}^{2}}, \\
\frac{\partial^{2} y}{\partial e_{3}^{2}} & =-\frac{\partial^{3} y}{\partial e_{1} \partial e_{2} \partial e_{3}}-e_{1} \cdot \frac{\partial^{3} y}{\partial e_{2}^{2} \partial e_{3}}-e_{2} \cdot \frac{\partial^{3} y}{\partial e_{2} \partial e_{3}^{2}}-e_{3} \cdot \frac{\partial^{3} y}{\partial e_{3}^{3}}-\frac{\partial^{2} y}{\partial e_{3}^{2}}
\end{aligned}
$$

Solving for the second-order partials, we get the following formulae. In the notation of Theorem 5.2,

$$
\begin{aligned}
\partial_{2, w} & =\partial_{\ell, w}=\sum_{\sigma=0}^{n \cdot(\varpi-\ell)} f_{\sigma} \cdot \partial_{\varpi, \sigma+w}=\sum_{\sigma=0}^{3} f_{\sigma} \cdot \partial_{\varpi, \sigma+w} \quad \text { for } w \in\{3,4,5,6\}, \\
\frac{\partial^{2} y}{\partial e_{1} \partial e_{2}} & =\frac{1}{2}\left(-\frac{\partial^{3} y}{\partial e_{1}^{3}}-e_{1} \cdot \frac{\partial^{3} y}{\partial e_{1}^{2} \partial e_{2}}-e_{2} \cdot \frac{\partial^{3} y}{\partial e_{1} \partial e_{2}^{2}}-e_{3} \cdot \frac{\partial^{3} y}{\partial e_{1} \partial e_{2} \partial e_{3}}\right), \\
\frac{\partial^{2} y}{\partial e_{2}^{2}} & =\frac{1}{2}\left(-\frac{\partial^{3} y}{\partial e_{1}^{2} \partial e_{2}}-e_{1} \cdot \frac{\partial^{3} y}{\partial e_{1} \partial e_{2}^{2}}-e_{2} \cdot \frac{\partial^{3} y}{\partial e_{2}^{3}}-e_{3} \cdot \frac{\partial^{3} y}{\partial e_{2}^{2} \partial e_{3}}\right), \\
\frac{\partial^{2} y}{\partial e_{2} \partial e_{3}} & =\frac{1}{2}\left(-\frac{\partial^{3} y}{\partial e_{1}^{2} \partial e_{3}}-e_{1} \cdot \frac{\partial^{3} y}{\partial e_{1} \partial e_{2} \partial e_{3}}-e_{2} \cdot \frac{\partial^{3} y}{\partial e_{2}^{2} \partial e_{3}}-e_{3} \cdot \frac{\partial^{3} y}{\partial e_{2} \partial e_{3}^{2}}\right), \\
\frac{\partial^{2} y}{\partial e_{3}^{2}} & =\frac{1}{2}\left(-\frac{\partial^{3} y}{\partial e_{1} \partial e_{2} \partial e_{3}}-e_{1} \cdot \frac{\partial^{3} y}{\partial e_{2}^{2} \partial e_{3}}-e_{2} \cdot \frac{\partial^{3} y}{\partial e_{2} \partial e_{3}^{2}}-e_{3} \cdot \frac{\partial^{3} y}{\partial e_{3}^{3}}\right) .
\end{aligned}
$$

If we substitute (6.14), (6.15), and (6.16) into (6.7) for the second-order partial derivatives, we get

$$
\begin{aligned}
\frac{\partial y}{\partial e_{2}}= & -\frac{\partial^{2} y}{\partial e_{1}^{2}}+\frac{1}{2} e_{1} \frac{\partial^{3} y}{\partial e_{1}^{3}}+\frac{1}{2}\left(e_{1}^{2}+e_{2}\right) \frac{\partial^{3} y}{\partial e_{1}^{2} \partial e_{2}}+\frac{1}{2}\left(2 e_{1} e_{2}+e_{3}\right) \frac{\partial^{3} y}{\partial e_{1} \partial e_{2}^{2}} \\
& +\frac{1}{2}\left(2 e_{1} e_{3}+e_{2}^{2}\right) \frac{\partial^{3} y}{\partial e_{2}^{3}}+e_{2} e_{3} \cdot \frac{\partial^{3} y}{\partial e_{2}^{2} \partial e_{3}}+e_{3}^{2} \frac{\partial^{3} y}{\partial e_{2} \partial e_{3}^{2}} .
\end{aligned}
$$

If we substitute (6.14), (6.15), (6.16), and (6.17) into (6.8) for the second-order partial derivatives, we get

$$
\begin{aligned}
\frac{\partial y}{\partial e_{3}}= & \frac{1}{2} \frac{\partial^{3} y}{\partial e_{1}^{3}}+e_{1} \frac{\partial^{3} y}{\partial e_{1}^{2} \partial e_{2}}+\frac{1}{2}\left(e_{1}^{2}+2 e_{2}\right) \frac{\partial^{3} y}{\partial e_{1} \partial e_{2}^{2}}+\left(e_{1} e_{2}+e_{3}\right) \frac{\partial^{3} y}{\partial e_{2}^{3}} \\
& +\frac{1}{2}\left(2 e_{1} e_{3}+e_{2}^{2}\right) \cdot \frac{\partial^{3} y}{\partial e_{2}^{2} \partial e_{3}}+e_{2} e_{3} \frac{\partial^{3} y}{\partial e_{2} \partial e_{3}^{2}}+\frac{1}{2} e_{3}^{2} \frac{\partial^{3} y}{\partial e_{3}^{3}} .
\end{aligned}
$$


Now substitute (6.18) and (6.19) for the first-order partial derivatives and (6.14), (6.15), (6.16), and (6.17) for the second-order partial derivatives into equation (6.4), and combine terms to get

$$
\begin{aligned}
D^{2} y= & e_{1}^{\prime \prime} \cdot \frac{\partial y}{\partial e_{1}}+\left(\left(e_{1}^{\prime}\right)^{2}-e_{2}^{\prime \prime}\right) \frac{\partial^{2} y}{\partial e_{1}^{2}}+\left(-e_{1}^{\prime} e_{2}^{\prime}+\frac{1}{2} e_{1} e_{2}^{\prime \prime}+\frac{1}{2} e_{3}^{\prime \prime}\right) \frac{\partial^{3} y}{\partial e_{1}^{3}} \\
+ & \left(-e_{1} e_{1}^{\prime} e_{2}^{\prime}-e_{1}^{\prime} e_{3}^{\prime}-\frac{1}{2}\left(e_{2}^{\prime}\right)^{2}+e_{1} e_{3}^{\prime \prime}+\frac{1}{2} e_{1}^{2} e_{2}^{\prime \prime}+\frac{1}{2} e_{2} e_{2}^{\prime \prime}\right) \frac{\partial^{3} y}{\partial e_{1}^{2} \partial e_{2}} \\
+ & \left(-e_{2} e_{1}^{\prime} e_{2}^{\prime}-e_{1} e_{1}^{\prime} e_{3}^{\prime}-\frac{1}{2} e_{1}\left(e_{2}^{\prime}\right)^{2}-e_{2}^{\prime} e_{3}^{\prime}+e_{1} e_{2} e_{2}^{\prime \prime}+\frac{1}{2} e_{3} e_{2}^{\prime \prime}+\frac{1}{2} e_{1}^{2} e_{3}^{\prime \prime}+e_{2} e_{3}^{\prime \prime}\right) \frac{\partial^{3} y}{\partial e_{1} \partial e_{2}^{2}} \\
+ & \left(-e_{3} e_{1}^{\prime} e_{2}^{\prime}-e_{2} e_{1}^{\prime} e_{3}^{\prime}-\frac{1}{2} e_{2}\left(e_{2}^{\prime}\right)^{2}-e_{1} e_{2}^{\prime} e_{3}^{\prime}-\frac{1}{2}\left(e_{3}^{\prime}\right)^{2}\right. \\
& \left.+e_{1} e_{3} e_{2}^{\prime \prime}+\frac{1}{2} e_{2}^{2} e_{2}^{\prime \prime}+e_{1} e_{2} e_{3}^{\prime \prime}+e_{3} e_{3}^{\prime \prime}\right) \frac{\partial^{3} y}{\partial e_{2}^{3}} \\
+ & \left(-e_{3} e_{1}^{\prime} e_{3}^{\prime}-\frac{1}{2} e_{3} \cdot\left(e_{2}^{\prime}\right)^{2}-e_{2} e_{2}^{\prime} e_{3}^{\prime}-\frac{1}{2} e_{1}\left(e_{3}^{\prime}\right)^{2}+e_{2} e_{3} e_{2}^{\prime \prime}+e_{1} e_{3} e_{3}^{\prime \prime}+\frac{1}{2} e_{2}^{2} e_{3}^{\prime \prime}\right) \frac{\partial^{3} y}{\partial e_{2}^{2} \partial e_{3}} \\
+ & \left(-e_{3} e_{2}^{\prime} e_{3}^{\prime}-\frac{1}{2} e_{2}\left(e_{3}^{\prime}\right)^{2}+\frac{1}{2} e_{3}^{2} e_{2}^{\prime \prime}+e_{2} e_{3} e_{3}^{\prime \prime}\right) \frac{\partial^{3} y}{\partial e_{2} \partial e_{3}^{2}}+\left(\frac{1}{2} e_{3}^{2} e_{3}^{\prime \prime}-\frac{1}{2} e_{3}\left(e_{3}^{\prime}\right)^{2}\right) \frac{\partial^{3} y}{\partial e_{3}^{3}}
\end{aligned}
$$

If we now specialize $\alpha$ to a positive integer $q$ and sum over the three roots of $P$, we may replace $y$ with the $q$ th powersum $p_{q}$. With Mathematica one can easily recursively compute the first twenty powersums as polynomials of $e_{1}, e_{2}$, and $e_{3}$. One can then easily verify that this partial differential resolvent holds for these powersums. In the notation of Theorem 5.2, $\tau_{(0,2) \times 1}=e_{1}^{\prime \prime}, \tau_{(0,2) \times 2}=\left(e_{1}^{\prime}\right)^{2}-e_{2}^{\prime \prime}, \ldots, \tau_{(0,2) \times 9}=(1 / 2) e_{3}^{2} e_{3}^{\prime \prime}-$ $(1 / 2) e_{3}\left(e_{3}^{\prime}\right)^{2}$.

7. Trivial factorization of the powersum formula. Now we return to the problem of finding the Cohnian, $\sum_{m=0}^{n} \sum_{i=0}^{\Omega-m} \theta_{i, m} \cdot \alpha^{i} D^{m} y=0$, of a polynomial $P$ of degree $n$ whose roots are differentially independent over constants. By an easy extension of basic algebra to differential algebra (see [6, Theorem 1, page 23]) the roots of $P$ are differentially independent over constants if and only if the coefficients $e$ of $P$ are differentially independent over constants. So, we may refer interchangeably to either the roots or the coefficients of $P$ as being differentially independent over constants. Reformulating the notation in Section 1 , the set $S$ of indices of the nonzero coefficients in the Cohnian equals $\left\{(i, m) \ni i+m \in[\Omega], m \in[n]_{0}, i \geq 0\right\}$, where $\Omega=n \cdot(n-1) / 2+1$. So, by Definition 3.2 for $\Phi$ as the size of the set $S$ and for $\Psi$ as the dimension of the matrices appearing in the powersum formula (3.1), we see that $\Psi=n \cdot \Omega$ and $\Phi=n \cdot \Omega+1$.

We wish to present first a "trivial" factorization theorem. We refer to it as trivial because the determinant of the matrix that factors from the matrix $A_{i, m}$ in (3.1) has no weight if $q$ is taken to span the set [ $\Psi]$. In other words, this determinant is just an integer. 
THEOREM 7.1 (trivial factorization theorem). Fix a pair $(i, m) \in S$. Then the matrix, $\left[D^{m^{\prime}} q^{i^{\prime}} p_{q}\right]_{\left(i^{\prime}, m^{\prime}\right) \times q}$, with columns indexed by $q \in[\Psi]$ and rows indexed by $\left(i^{\prime}, m^{\prime}\right) \in S \ni$ $\left(i^{\prime}, m^{\prime}\right) \neq(i, m)$, factors over the differential ring, $\mathbb{Q}\{e\}$, as

$$
\begin{aligned}
& {\left[D^{m^{\prime}} q^{i^{\prime}} p_{q}\right]_{\left(i^{\prime}, m^{\prime}\right) \times q}}
\end{aligned}
$$

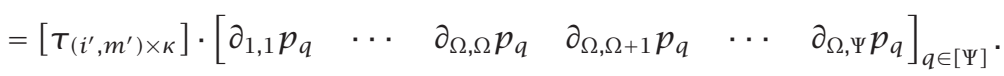

The factor $\left[\begin{array}{llllll}\partial_{1,1} p_{q} & \cdots & \partial_{\Omega, \Omega} p_{q} & \partial_{\Omega, \Omega+1} p_{q} & \cdots & \partial_{\Omega, \Psi} p_{q}\end{array}\right]_{q \in[\Psi]}$ is a $\Psi \times \Psi$ uppertriangular matrix with entries in $\mathbb{Z}\{e\}$. The factor $\left[\tau_{(i, m) \times \kappa}\right]$ is a $\Psi \times \Psi$ matrix with entries in $\mathbb{Q}\{e\}_{n}$.

Proof. By Theorem 5.2, we have $D^{m_{\Theta} i} p_{q}=\sum_{\kappa=1}^{\varpi} \tau_{(i, m) \times \kappa} \cdot \partial_{\kappa, \kappa} p_{q}+\sum_{\kappa=\varpi+1}^{n \cdot \varpi} \tau_{(i, m) \times \kappa}$. $\partial_{\varpi, \kappa} p_{q}$ with $\tau_{(i, m) \times \kappa} \in \mathbb{Q}\{e\}_{n}$. Setting $\varpi=\Omega$ gives us $D^{m} \Theta^{i} p_{q}=\sum_{\kappa=1}^{\Omega} \tau_{(i, m) \times \kappa} \cdot \partial_{\kappa, \kappa} p_{q}+$ $\sum_{\kappa=\Omega+1}^{\Psi} T_{(i, m) \times \kappa} \cdot \partial_{\Omega, \kappa} p_{q}$. So

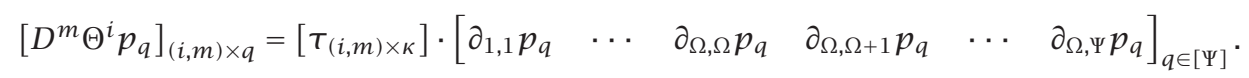

It is obvious that the entries $\partial_{\ell, w} p_{q}$ lie in $\mathbb{Z}[e]$.

In Theorem 8.1 we will be interested in an important variation of Theorem 7.1, in which we choose $\varpi=\Omega-1$ instead of $\varpi=\Omega$, and ignore the powersums. Define the $1 \times \Psi$ row vector, $\vec{p} \equiv\left[p_{q}\right]_{q \in[\Psi]}$, of powersums. In Theorem 8.1 we will define the $\Psi \times 1$ column vector of partial derivatives $\mathfrak{A}_{\Omega} \equiv\left\{\partial_{\ell, w} \ni \ell=w \in[\Omega]\right\} \bigcup\left\{\partial_{\Omega, w} \ni \Omega+1 \leq w \leq\right.$ $\Psi\}$.

DEFINITION 7.2. If $M_{\text {left }}$ is a matrix whose $(i, j)$ entry is $\sum_{\ell, w} h_{\ell, w}^{i, j} \cdot \partial_{\ell, w}$ with $h_{\ell, w}^{i, j} \in$ $\mathbb{Q}\{e\}$ and $M_{\text {right }}$ is a matrix whose $(j, k)$ entry is $g_{j, k} \in \mathbb{Z}[e]$, then the operator $*$ is defined such that $M_{\text {left }} * M_{\text {right }}$ is the matrix whose $(i, k)$ entry is $\sum_{j} \sum_{\ell, w} h_{\ell, w}^{i, j} \cdot \partial_{\ell, w} g_{j, k}$. Similarly, if $M_{\text {left }}$ is a matrix whose $(i, j)$ entry is $\sum_{u, v} h_{u, v}^{i, j} \cdot D^{u} \Theta^{v}$ with $h_{u, v}^{i, j} \in \mathbb{Q}\{e\}$ and $M_{\text {right }}$ is a matrix whose $(j, k)$ entry is $g_{j, k} \in \mathbb{Z}[e]$, then the operator $*$ is defined such that $M_{\text {left }} * M_{\text {right }}$ is the matrix whose $(i, k)$ entry is $\sum_{j} \sum_{u, v} h_{u, v}^{i, j} \cdot D^{u} \Theta^{v} g_{j, k}$.

Then we may write the right factor $\Psi \times \Psi$ matrix in (7.1)

$$
\left[\begin{array}{llllll}
\partial_{1,1} p_{q} & \cdots & \partial_{\Omega, \Omega} p_{q} & \partial_{\Omega, \Omega+1} p_{q} & \cdots & \partial_{\Omega, \Psi} p_{q}
\end{array}\right]_{q \in[\Psi]}
$$

as the product of a $\Psi \times 1$ with a $1 \times \Psi$ matrix $\mathcal{A}_{\Omega} * \vec{p}$. We will use this shorter notation as much as possible from now on. By Theorem 7.1, the term $\operatorname{det}\left(\mathcal{A}_{\Omega} * \vec{p}\right) \in \mathbb{Z}[e]$ factors out of $\operatorname{det}\left[D^{m} \Theta^{i} p_{q}\right]_{(i, m) \times q}$, leaving $\pm \operatorname{det}\left(\left[\boldsymbol{\tau}_{(i, m) \times \kappa}\right]\right) \in \mathbb{Q}\{e\}$ as the quotient. The isobaric weight of the entry $\partial_{\ell, w} p_{q}$ in $\mathcal{A}_{\Omega} * \vec{p}$ is $q-w$ if $q \geq w$ and $\partial_{\ell, w} p_{q}=0$ if $q<w$. When $q=$ $w$, it is well known that $\partial_{\ell, w} p_{q}=w ! \in \mathbb{N}$. Since $\mathcal{A}_{\Omega} * \vec{p}$ is upper-triangular, $\operatorname{det}\left(\mathcal{A}_{\Omega} * \vec{p}\right)$ is just the product of the entries down its main diagonal $\prod_{w=1}^{\Psi} w$ !.

The matrix, $\left[\boldsymbol{\tau}_{\left(i^{\prime}, m^{\prime}\right) \times k}\right]_{\left(i^{\prime}, m^{\prime}\right) \neq(i, m)}$, in Theorem 7.1 is the transition matrix expressing the operators, $D^{m} \Theta^{i}$, that appear in the powersum formula (3.1) in terms of the basis of $\Psi$ distinct partial derivatives $\mathcal{H}$. 
The reader might consider it unnecessary to use the powersums of the roots, and hence the powersum formula (3.1), to find the terms of a resolvent since the problem has been reduced to finding a linear combination of the operators, $D^{m} \Theta^{i}$, over $\mathbb{Z}\{e\}$. This is true in the case under consideration, when the roots are differentially independent over constants. However, the powersum formula (3.1) is indeed useful for finding resolvents of polynomials whose roots may bear unknown relations. Furthermore, even in the case when the roots are differentially independent over constants, it turns out that it is much easier and faster on a computer algebra system to compute total derivatives and partial derivatives of the powersums with respect to the polynomial coefficients, $e$, than to compute such derivatives of a transcendental element like $y$. As a demonstration, the author will use these powersums to compute the terms in a resolvent of a particular cubic polynomial in Section 12.

8. The main factorization theorem. We now wish to expand these ordinary derivative operators $D^{m} \Theta^{i}$ as sums of the partial derivative operators $\partial_{\ell, w}$ with respect to the coefficients $e$ of the polynomial $P$ given by Definition 1.1. First, we must count the number of distinct partial derivative operators $\partial_{\ell, w}$ needed to represent a particular ordinary derivative operator $D^{m} \Theta^{i}$ appearing effectively in the Cohnian.

TheOREM 8.1. For any $\varpi \in[\Omega]$, define $\boldsymbol{\kappa}_{\varpi} \equiv\left\{D^{m} \Theta^{i} \ni(i, m) \in S, i+m \in[\varpi]\right\}$ to be the subset of ordinary derivative operators $D^{m} \Theta^{i}$ corresponding to the derivative operators $\alpha^{i} D^{m}$ appearing effectively in the $\alpha$-resolvent $\sum_{m=0}^{n} \sum_{i=0}^{\Omega-m} F_{i, m} \cdot \alpha^{i} D^{m} y=0$ of $P$, constrained by $i+m \leq \varpi$. For this same $\varpi$, define $\mathcal{A}_{\varpi} \equiv\left\{\partial_{\ell, \ell}\right\}_{\ell=1}^{\ell=\varpi} \bigcup\left\{\partial_{\varpi, w}\right\}_{w=\varpi+1}^{w=n \cdot \varpi}$ to be the set of partial derivative operators needed to represent the derivative operators in $\aleph_{\varpi}$. Let $L$ denote the unique minimal positive integer such that $\left|\aleph_{L}\right|=\left|\mathfrak{A}_{L}\right|$. Then $L=n \cdot(n-1) / 2$.

Proof. By Theorem 5.2, for any $\varpi \geq i+m$, the operator $D^{m} \Theta^{i}$ can be expressed as a linear combination over $\mathbb{Q}\{e\}$ of the partial derivatives $\left\{\partial_{\ell, \ell}\right\}_{\ell=1}^{\ell=\varpi} \bigcup\left\{\partial_{\varpi, w}\right\}_{w=\varpi+1}^{w=n \cdot \varpi}$, a total of $n \cdot \varpi$ partial derivatives. Thus $\left|\mathcal{A}_{\varpi}\right|=n \cdot \varpi$. By directly counting the number of indices $(i, m)$ in the set $\kappa_{\varpi} \equiv\left\{D^{m} \Theta^{i} \ni(i, m) \in S, i+m \in[\varpi]\right\}$ we find that

$$
\left|\aleph_{\varpi}\right|=-1+\sum_{m=0}^{n}(\varpi-m+1)=n \cdot \varpi+\varpi-\frac{n \cdot(n-1)}{2},
$$

where the initial -1 accounts for the fact that $(0,0) \notin S$. Hence, $\left|\aleph_{\varpi}\right|=\left|\mathcal{A}_{\varpi}\right|$ if and only if $n \cdot \varpi=n \cdot \varpi+\varpi-n \cdot(n-1) / 2$ if and only if $\varpi=n \cdot(n-1) / 2$. So $L=n \cdot(n-1) / 2$.

Now give the elements of the sets $\aleph_{\varpi}$ and $\mathcal{A}_{\varpi}$ defined in the proof of Theorem 8.1 an ordering. Using these definitions of $\aleph_{\varpi}$ and $\mathcal{A}_{\varpi}$, we will consider the following three vectors in Theorem 8.2: the $(n \cdot L) \times 1$ vector

$$
\aleph_{L}=\left\{D^{m} \Theta^{i} \ni(i, m) \in S, i+m \in[L]\right\},
$$

the $(n \cdot L) \times 1$ vector

$$
\mathcal{A}_{L}=\left\{\partial_{\ell, w} \ni w=\ell \in[L]\right\} \bigcup\left\{\partial_{\ell, w} \ni \ell=L, w \in[n \cdot L] \ni w>L\right\},
$$


and the $\Psi \times 1$ vector

$$
\mathfrak{A}_{\Omega}=\left\{\partial_{\ell, w} \ni w=\ell \in[\Omega]\right\} \bigcup\left\{\partial_{\ell, w} \ni \ell=\Omega, w \in[n \cdot \Omega] \ni w>\Omega\right\} .
$$

We remind the reader that $S=\left\{(i, m) \ni i+m \in[\Omega], m \in[n]_{0}, i \geq 0\right\}$. Let $S_{L} \subset S$ denote the subset of $S, S_{L} \equiv\left\{(i, m) \ni i+m \in[L], m \in[n]_{0}, i \geq 0\right\}$.

Setting $\varpi=L$ in Theorem 5.2 gives us $D^{m} \Theta^{i}=\sum_{K=1}^{L} \hat{\boldsymbol{\tau}}_{(i, m) \times K} \cdot \partial_{\kappa, K}+\sum_{\kappa=\Omega}^{n \cdot L} \hat{\boldsymbol{\tau}}_{(i, m) \times \kappa}$. $\partial_{L, \kappa}$ for some $\hat{\boldsymbol{\tau}}_{(i, m) \times \kappa} \in \mathbb{Q}\{e\}$. Define the $n \cdot L \times n \cdot L$ transition matrix with entries in $\mathbb{Z}\{e\}$

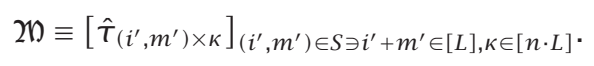

Then $2 \mathfrak{b}$ is the transition matrix, $2 \mathfrak{b}: \mathfrak{A}_{L} \rightarrow \mathfrak{\aleph}_{L}$, from the vector of partial derivatives $\mathfrak{A}_{L}$ given by (8.3) to the vector of ordinary derivatives $\aleph_{L}$ given by (8.2). So we may write $\aleph_{L}=2 \mathfrak{b} \cdot \mathfrak{A}_{L}$. For future reference, we note that $\aleph_{\Omega}$ is simply the complete set of $\Phi$ operators, $D^{m} \Theta^{i}$, appearing in the resolvent. Theorem 8.1 guarantees that $2 \mathrm{~b}$ is a square matrix, and therefore $\operatorname{det} 2 \mathfrak{b}$ is defined.

THEOREM 8.2 (main factorization theorem). Let $P(t) \equiv \sum_{i=0}^{n}(-1)^{n-i} e_{n-i} \cdot t^{i}$ be $a$ monic polynomial whose coefficients $\left\{e_{i}\right\}_{i=1}^{n}$ are differentially independent over $\mathbb{Z}$ with respect to the derivation $D$. Let $\sum_{m=0}^{n} \sum_{i=0}^{\Omega-m} F_{i, m} \cdot \alpha^{i} D^{m} y=0, F_{0,0}=0$, be the differential resolvent of $P$ given by the powersum formula (3.1). Let $2 \mathrm{~b}$ be defined by (8.5). Let $S \subset S$ denote the subset of $2 n+2$ terms in the resolvent, $\{(0, m)\}_{m=1}^{n} \cup\{(1,0)\} \cup\{(i, m) \ni i+$ $m=\Omega\}_{m=0}^{n}$. Then a common factor of $F_{i, m}$, for each $(i, m) \in \underset{\sim}{S}$, is the determinant of $2 n$. In other words, for each $(i, m) \in S$, det2n divides $F_{i, m}$ in the ring $\mathbb{Q}\{e\}$.

Proof. Fix $(i, m) \in S$. Let $\vec{p} \equiv\left(p_{1}, \ldots, p_{\Psi}\right)$ denote the $1 \times \Psi$ row vector of the first $\Psi$ powersums.

By partial differential resolvent (5.3) with $\varpi=L$, we may express any $\partial_{\ell, w}$ with $w \leq$ $\Psi$ as a linear combination over $\mathbb{Q}[e]$ of $\partial_{\ell^{\prime}, w^{\prime}} \in \mathcal{A}_{\Omega}$. In other words, there exists an $n \cdot L \times \Psi$ transition matrix, $\mathrm{N}: \mathfrak{A}_{\Omega} \rightarrow \mathfrak{A}_{L}$, with entries in $\mathbb{Q}[e]$, such that $\mathfrak{A}_{L}=\mathrm{N} * \mathfrak{A}_{\Omega}$. Then $2 \mathfrak{b} * \mathrm{~N}: \mathfrak{A}_{\Omega} \rightarrow \mathrm{N}_{L}$, so $\mathrm{N}_{L}=(2 \mathfrak{b} * \mathrm{~N}) * \mathfrak{A}_{\Omega}$. Let $\Lambda_{i, m}$ denote the $\Psi \times 1$ vector of operators, $\left\{D^{m^{\prime}} \Theta^{i^{\prime}} \ni\left(i^{\prime}, m^{\prime}\right) \in S,\left(i^{\prime}, m^{\prime}\right) \neq(i, m)\right\}$, appearing in the powersum formula (3.1).

CASE 1. $(i, m) \in S \ni i+m=\Omega$.

In this case $\Lambda_{i, m}=\left[\begin{array}{c}\mathrm{N}_{L} \\ \mathbb{R}_{i, m}\end{array}\right]$, where $\mathbb{R}_{i, m}=\left\{D^{m^{\prime}} \Theta^{\Omega-m^{\prime}}, m^{\prime} \in[n]_{0}, m^{\prime} \neq m\right\}$ is the column of $n$ operators obtained from $\Lambda_{i, m}$ by removing $\aleph_{L}$. Let $\mathrm{T}_{i, m}$ be the $n \times \Psi$ transition matrix consisting of entries in $\mathbb{Z}\{e\}$ from $\mathcal{A}_{\Omega}$ to the set of $n$ operators $\mathbb{R}_{i, m}$. So $\mathbb{R}_{i, m}=\mathrm{T}_{i, m} * \mathfrak{A}_{\Omega}$. Then $\Lambda_{i, m}=\left[\begin{array}{c}2 \mathrm{2b} * \mathrm{~N} * \mathcal{A}_{\Omega} \\ \mathrm{T}_{i, m} * \mathcal{H}_{\Omega}\end{array}\right]=\left[\begin{array}{c}2 \mathrm{2n} * \mathrm{~N} \\ \mathrm{~T}_{i, m}\end{array}\right] * \mathcal{H}_{\Omega}=\left[\begin{array}{cc}2 b & 0 \\ 0 & \mathrm{I}_{n}\end{array}\right] *\left[\begin{array}{c}\mathrm{N} \\ \mathrm{T}_{i, m}\end{array}\right] * \mathfrak{A}_{\Omega}$. So by (3.1) $\mathrm{A}_{i, m}=\Lambda_{i, m} * \vec{p}$ and $F_{i, m}=\operatorname{det} \mathrm{A}_{i, m}=\operatorname{det}\left(\left[\begin{array}{cc}2 n & 0 \\ 0 & \mathrm{I}_{n}\end{array}\right] *\left[\begin{array}{c}\mathrm{N} \\ \mathrm{T}_{i, m}\end{array}\right] *\left(\mathfrak{A}_{\Omega} * \vec{p}\right)\right)=\operatorname{det}(2 \mathfrak{b})$. $\left.\operatorname{det}\left[\begin{array}{c}\mathrm{N} \\ \mathrm{T}_{i, m}\end{array}\right]\right) \cdot \operatorname{det}\left(\mathcal{A}_{\Omega} * \vec{p}\right)$ after a suitable ordering of the rows of $\mathrm{T}_{i, m}$.

CASE 2. $(i, m) \in S \ni i=0$.

Define $\kappa_{L} \Theta \equiv\left\{D^{m^{\prime}} \Theta^{i^{\prime}} \Theta \ni D^{m^{\prime}} \Theta^{i^{\prime}} \in \mathcal{N}_{L}\right\}=\left\{D^{m^{\prime}} \Theta^{i^{\prime}+1} \ni\left(i^{\prime}, m^{\prime}\right) \in S, i^{\prime}+m^{\prime} \in[L]\right\}$. Then $\Lambda_{i, m}=\left[\begin{array}{c}\kappa_{L} \Theta \\ \mathbb{R}_{i, m}\end{array}\right]$, where $\mathbb{R}_{i, m}$ is the column of $n$ operators with entries taken from 
the vector $\left\{D^{m^{\prime}}, m^{\prime} \in[n], m^{\prime} \neq m\right\} \bigcup\{\Theta\}$ which were obtained from $\Lambda_{i, m}$ by removing $\aleph_{L} \Theta$. We have $\kappa_{L} \Theta=2 \mathfrak{W} * \mathcal{H}_{L} \Theta$, where $\mathcal{H}_{L} \Theta \equiv\left\{\partial_{\ell, w} \Theta \ni \partial_{\ell, w} \in P\right\}$ is an $n \cdot L \times 1$ column vector of partial derivative operators. Since the order of the derivative operators in $\mathfrak{A}_{L} \Theta$ is less than or equal to $L+1=\Omega$, we know there exists an $n \cdot L \times \Psi$ transition matrix $\tilde{\mathrm{N}}: \mathfrak{A}_{\Omega} \rightarrow \mathcal{H}_{L} \Theta$ with entries in $\mathbb{Q}[e]$, such that $\mathfrak{A}_{L} \Theta=\tilde{\mathrm{N}} * \mathcal{H}_{\Omega}$. So $\mathfrak{\kappa}_{L} \Theta=(\mathfrak{2} \mathrm{W} * \tilde{\mathrm{N}}) * \mathcal{A}_{\Omega}$. Let $\mathrm{T}_{i, m}$ be the $n \times \Psi$ transition matrix consisting of entries in $\mathbb{Z}\{e\}$ from $\mathcal{A}_{\Omega}$ to the set of $n$ operators $\mathbb{R}_{i, m}$. So $\mathbb{R}_{i, m}=\mathrm{T}_{i, m} * \mathcal{A}_{\Omega}$. Then

$$
\Lambda_{i, m}=\left[\begin{array}{c}
2 \mathfrak{W} * \tilde{\mathrm{N}} \\
\mathrm{T}_{i, m}
\end{array}\right] * \mathcal{H}_{\Omega}
$$

So $F_{i, m}=\operatorname{det} \mathrm{A}_{i, m}=\operatorname{det}\left(\left[\begin{array}{cc}2 n & 0 \\ 0 & \mathrm{I}_{n}\end{array}\right] *\left[\begin{array}{c}\tilde{\mathrm{N}} \\ \mathrm{T}_{i, m}\end{array}\right] *\left(\mathcal{A}_{\Omega} * \vec{p}\right)\right)=\operatorname{det}(2 \mathfrak{W}) \cdot \operatorname{det}\left[\begin{array}{c}\tilde{\mathrm{N}} \\ \mathrm{T}_{i, m}\end{array}\right] \cdot \operatorname{det}\left(\mathcal{A}_{\Omega} * \vec{p}\right)$ after a suitable ordering of the rows of $\mathrm{T}_{i, m}$.

CASE 3. $(i, m)=(1,0) \in S$.

We have $\Lambda_{i, m}=\left[\begin{array}{l}\kappa_{L} \Theta \\ \mathbb{R}_{i, m}\end{array}\right]$, where $\mathbb{R}_{i, m}=\left(D^{m^{\prime}}\right)_{m^{\prime}=1}^{n}$. The matrix algebra is the same as in Case 2. So $\operatorname{det} 2 \mathfrak{W}$ divides $F_{i, m}$ in $\mathbb{Q}\{e\}$ for all $(i, m) \in S$.

REMARKS 8.3. Theorem 8.2 is [6, Theorem 62, page 114]. Observe that the determinant of the matrix $2 \mathfrak{W}$ has weight $\sum_{\kappa=1}^{n \cdot L} \kappa=n \cdot L(n \cdot L+1) / 2$. Therefore, the weight of $F_{i, m} \div \operatorname{det} 2 \mathfrak{h}$ equals

$$
\frac{\Psi(\Psi+1)}{2}-\frac{n \cdot L(n \cdot L+1)}{2}=n \cdot \frac{n^{3}-n^{2}+n+1}{2} .
$$

Therefore, Theorem 8.2 has reduced the weight for those terms of the resolvent indexed by $(i, m) \in S$ from sextic in $n$ to quartic in $n$.

\section{Quadratic resolvent by the powersum formula}

EXAMPLE 9.1. Let $P(t) \equiv t^{2}-e_{1} \cdot t+e_{2}$ be a quadratic polynomial whose coefficients $e_{1}$ and $e_{2}$ are differentially independent over $\mathbb{Q}$. So $n=2, L=n \cdot(n-1) / 2=1, \Omega=$ $L+1=2, \Psi=n \cdot \Omega=4$, and $\Phi=1+\Psi=5$. The discriminant of $P$ is $\Delta=e_{1}^{2}-4 e_{2}$. Later we will let the prime ' denote differentiation by $D$. The first four powersums are given in terms of the elementary symmetric functions by $p_{1}=e_{1}, p_{2}=e_{1}^{2}-2 e_{2}, p_{3}=e_{1}^{3}-3 e_{1} e_{2}$, and $p_{4}=e_{1}^{4}-4 e_{1}^{2} e_{2}+2 e_{2}^{2}$. Define

$$
\vec{p} \equiv\left[\begin{array}{llll}
p_{1} & p_{2} & p_{3} & p_{4}
\end{array}\right]
$$

Theorem 8.2 allows us to factor those terms of the resolvent indexed by $S=\{(0,1)$, $(0,2),(1,0),(1,1),(2,0)\}$. Thus, for the quadratic, $S=S$, so all the terms factor by Theorem 8.2. The form of the Cohnian is given by (4.1) as

$$
\theta_{0,2} \cdot D^{2} y+\left(\theta_{0,1}+\theta_{1,1} \cdot \alpha\right) \cdot D y+\left(\theta_{1,0} \cdot \alpha+\theta_{2,0} \cdot \alpha^{2}\right) \cdot y=0
$$

First we will show how the powersum formula (3.1) works. Then we will factor it with the help of Theorem 8.2. 
Specializing $\alpha$ to the integers $[\Psi]=\{1,2,3,4\}$ in this resolvent and summing each of the resulting four equations over the two roots yields the system of four equations

$$
\begin{aligned}
& \theta_{0,2} \cdot D^{2} p_{1}+\left(\theta_{0,1}+\theta_{1,1} \cdot 1\right) \cdot D p_{1}+\left(\theta_{1,0} \cdot 1+\theta_{2,0} \cdot 1^{2}\right) \cdot p_{1}=0 \\
& \theta_{0,2} \cdot D^{2} p_{2}+\left(\theta_{0,1}+\theta_{1,1} \cdot 2\right) \cdot D p_{2}+\left(\theta_{1,0} \cdot 2+\theta_{2,0} \cdot 2^{2}\right) \cdot p_{2}=0 \\
& \theta_{0,2} \cdot D^{2} p_{3}+\left(\theta_{0,1}+\theta_{1,1} \cdot 3\right) \cdot D p_{3}+\left(\theta_{1,0} \cdot 3+\theta_{2,0} \cdot 3^{2}\right) \cdot p_{3}=0 \\
& \theta_{0,2} \cdot D^{2} p_{4}+\left(\theta_{0,1}+\theta_{1,1} \cdot 4\right) \cdot D p_{4}+\left(\theta_{1,0} \cdot 4+\theta_{2,0} \cdot 4^{2}\right) \cdot p_{4}=0
\end{aligned}
$$

This is a system of four homogeneous linear equations in the five variables, $\theta_{i, m}$. We may now solve for the terms $\theta_{i, m}$. First set $F_{i, m}$ to be the appropriate cofactor of the $4 \times 5$ matrix $\left[D^{m^{\prime}} q^{i^{\prime}} p_{q}\right]_{\substack{\left.i^{\prime}, m^{\prime}\right) \in S \\ q \in[4]}}$. This yields the powersum formula (3.1) for $F_{i, m}$. Then factor each $F_{i, m}$ over $\mathbb{Z}\{e\}$ to get $\theta_{i, m}$ such that the only factor common to all five $\theta_{i, m}$ is \pm 1 . By (3.1),

$$
\begin{gathered}
A_{0,2} \equiv\left[\begin{array}{llll}
D p_{1} & 1 \cdot D p_{1} & 1 p_{1} & 1^{2} p_{1} \\
D p_{2} & 2 \cdot D p_{2} & 2 p_{2} & 2^{2} p_{2} \\
D p_{3} & 3 \cdot D p_{3} & 3 p_{3} & 3^{2} p_{3} \\
D p_{4} & 4 \cdot D p_{4} & 4 p_{4} & 4^{2} p_{4}
\end{array}\right], \\
A_{0,1} \equiv\left[\begin{array}{llll}
D^{2} p_{1} & 1 \cdot D p_{1} & 1 p_{1} & 1^{2} p_{1} \\
D^{2} p_{2} & 2 \cdot D p_{2} & 2 p_{2} & 2^{2} p_{2} \\
D^{2} p_{3} & 3 \cdot D p_{3} & 3 p_{3} & 3^{2} p_{3} \\
D^{2} p_{4} & 4 \cdot D p_{4} & 4 p_{4} & 4^{2} p_{4}
\end{array}\right], \quad A_{1,1} \equiv\left[\begin{array}{lllll}
D^{2} p_{1} & D p_{1} & 1 p_{1} & 1^{2} p_{1} \\
D^{2} p_{2} & D p_{2} & 2 p_{2} & 2^{2} p_{2} \\
D^{2} p_{3} & D p_{3} & 3 p_{3} & 3^{2} p_{3} \\
D^{2} p_{4} & D p_{4} & 4 p_{4} & 4^{2} p_{4}
\end{array}\right], \\
A_{1,0} \equiv\left[\begin{array}{lllllll}
D^{2} p_{1} & D p_{1} & 1 \cdot D p_{1} & 1^{2} p_{1} \\
D^{2} p_{2} & D p_{2} & 2 \cdot D p_{2} & 2^{2} p_{2} \\
D^{2} p_{3} & D p_{3} & 3 \cdot D p_{3} & 3^{2} p_{3} \\
D^{2} p_{4} & D p_{4} & 4 \cdot D p_{4} & 4^{2} p_{4}
\end{array}\right], \quad A_{2,0} \equiv\left[\begin{array}{lllll}
D^{2} p_{1} & D p_{1} & 1 \cdot D p_{1} & 1 p_{1} \\
D^{2} p_{2} & D p_{2} & 2 \cdot D p_{2} & 2 p_{2} \\
D^{2} p_{3} & D p_{3} & 3 \cdot D p_{3} & 3 p_{3} \\
D^{2} p_{4} & D p_{4} & 4 \cdot D p_{4} & 4 p_{4}
\end{array}\right] .
\end{gathered}
$$

Then the powersum formula (3.1) gives $F_{0,2}=\operatorname{det} A_{0,2}, F_{0,1}=-\operatorname{det} A_{0,1}, F_{1,1}=\operatorname{det} A_{1,1}$, $F_{1,0}=-\operatorname{det} A_{1,0}$, and $F_{2,0}=\operatorname{det} A_{2,0}$. We have kept the columns of the matrices $A_{i, m}$ in the same order as the corresponding terms in the Cohnian, namely, $D^{2} \succ D \succ \alpha D \succ \alpha \succ \alpha^{2}$. We have also indexed the rows of $A_{i, m}$ by $q$, where $q$ is the weight of the powersum $p_{q}$, in order for the reader to see more clearly how the powersum formula works. However, for reasons that will become apparent later, we will prefer to work with a new matrix $\mathrm{A}_{i, m}$, rather than with $A_{i, m}$, where $\mathrm{A}_{i, m}$ is the transpose of $A_{i, m}$ after the columns of $A_{i, m}$ have been permuted a certain way.

By Theorem 8.2, $\mathfrak{A}_{L}$ is the $n \cdot L \times 1=2 \times 1$ vector of partial derivatives $\left[\begin{array}{l}\partial_{1,1} \\ \partial_{1,2}\end{array}\right]$, where $\partial_{1,1}=\partial / \partial e_{1}$ and $\partial_{1,2}=\partial / \partial e_{2}, \aleph_{L}$ is the $n \cdot L \times 1=2 \times 1$ vector $\left[\begin{array}{l}D \\ \Theta\end{array}\right]$, and $2 \mathfrak{W}$ is the $2 \times 2$ 
transition matrix from $\mathfrak{A}_{L}$ to $\aleph_{L} . \mathfrak{A}_{\Omega}$ is the $\Psi \times 1=4 \times 1$ vector of partial derivatives

$$
\left[\begin{array}{l}
\partial_{1,1} \\
\partial_{2,2} \\
\partial_{2,3} \\
\partial_{2,4}
\end{array}\right] \text {, where } \partial_{2,2}=\frac{\partial^{2}}{\partial e_{1}^{2}}, \partial_{2,3}=\frac{\partial^{2}}{\partial e_{1} \partial e_{2}}, \partial_{2,4}=\frac{\partial^{2}}{\partial e_{2}^{2}}
$$

By Definition 5.1 we have $\Theta=1 \cdot e_{1} \cdot\left(\partial / \partial e_{1}\right)+2 \cdot e_{2} \cdot\left(\partial / \partial e_{2}\right)=e_{1} \cdot \partial_{1,1}+2 \cdot e_{2} \cdot \partial_{1,2}$ and $D=e_{1}^{\prime}\left(\partial / \partial e_{1}\right)+e_{2}^{\prime}\left(\partial / \partial e_{2}\right)=e_{1}^{\prime} \partial_{1,1}+e_{2}^{\prime} \partial_{1,2}$ when acting on $\mathbb{Q}\left(e_{1}, e_{2}\right)$. Thus $2 \mathrm{~W}=\left[\begin{array}{cc}e_{1}^{\prime} & e_{2}^{\prime} \\ 1 \cdot e_{1} & 2 \cdot e_{2}\end{array}\right]$ since $2 \mathfrak{W} * \mathfrak{H}_{L}=\left[\begin{array}{cc}e_{1}^{\prime} & e_{2}^{\prime} \\ 1 \cdot e_{1} & 2 \cdot e_{2}\end{array}\right] *\left[\begin{array}{l}\partial_{1,1} \\ \partial_{1,2}\end{array}\right]=\left[\begin{array}{c}D \\ \Theta\end{array}\right]=\aleph_{L}$.

10. Expansion of the partial derivatives for the quadratic. In order to compute the various compositions of $D$ and $\Theta$, we note that $\partial_{i, j} \partial_{k, l}=\partial_{i+k, j+l}$, so $\partial_{1,1} \partial_{1,1}=\partial_{2,2}$, $\partial_{1,1} \partial_{1,2}=\partial_{2,3}$, and $\partial_{1,2} \partial_{1,2}=\partial_{2,4}$. We need to express the partial derivatives comprising $\mathfrak{A}_{L}$ in terms of the partial derivatives comprising $\mathcal{A}_{\Omega}$. The only partial derivative not in common between $\mathfrak{A}_{L}$ and $\mathfrak{A}_{\Omega}$ is $\partial_{1,2}$, so this is the only partial derivative that needs to be expressed in terms of the others. Choosing $j=2$ in partial differential resolvent (5.2), which corresponds to $\ell=1, w=2$, and $\varpi=2$ in (5.3), we get

$$
\partial_{1,2}=-\left(\partial_{2,2}+e_{1} \cdot \partial_{2,3}+e_{2} \cdot \partial_{2,4}\right)
$$

We may now compute the $n \cdot L \times \Psi=2 \times 4$ transition matrix $\mathrm{N}$ from $\mathfrak{A}_{\Omega}$ to $\mathfrak{A}_{L}$ such that $\mathfrak{A}_{L}=\mathrm{N} * \mathfrak{A}_{\Omega}$. We find that

$$
\left[\begin{array}{l}
\partial_{1,1} \\
\partial_{1,2}
\end{array}\right]=\left[\begin{array}{cccc}
1 & 0 & 0 & 0 \\
0 & -1 & -e_{1} & -e_{2}
\end{array}\right] *\left[\begin{array}{l}
\partial_{1,1} \\
\partial_{2,2} \\
\partial_{2,3} \\
\partial_{2,4}
\end{array}\right]
$$

so

$$
\mathrm{N}=\left[\begin{array}{cccc}
1 & 0 & 0 & 0 \\
0 & -1 & -e_{1} & -e_{2}
\end{array}\right]
$$

We may also compute the $n \cdot L \times \Psi=2 \times 4$ transition matrix $\tilde{N}$ from $\mathcal{A}_{\Omega}$ to $\mathcal{A}_{L} \Theta$ such that $\mathfrak{A}_{L} \Theta=\tilde{\mathrm{N}} * \mathcal{H}_{\Omega}$. We have

$$
\mathfrak{A}_{L} \Theta=\left[\begin{array}{c}
\partial_{1,1} \Theta \\
\partial_{1,2} \Theta
\end{array}\right]=\left[\begin{array}{l}
\partial_{1,1}\left(e_{1} \partial_{1,1}+2 e_{2} \partial_{1,2}\right) \\
\partial_{1,2}\left(e_{1} \partial_{1,1}+2 e_{2} \partial_{1,2}\right)
\end{array}\right]=\left[\begin{array}{c}
\partial_{1,1}+e_{1} \partial_{2,2}+2 e_{2} \partial_{2,3} \\
e_{1} \partial_{2,3}+2 \partial_{1,2}+2 e_{2} \partial_{2,4}
\end{array}\right] .
$$

So

$$
\mathcal{A}_{L} \Theta=\left[\begin{array}{c}
\partial_{1,1}+e_{1} \partial_{2,2}+2 e_{2} \partial_{2,3} \\
e_{1} \partial_{2,3}-2\left(\partial_{2,2}+e_{1} \cdot \partial_{2,3}+e_{2} \cdot \partial_{2,4}\right)+2 e_{2} \partial_{2,4}
\end{array}\right]=\left[\begin{array}{c}
\partial_{1,1}+e_{1} \cdot \partial_{2,2}+2 e_{2} \cdot \partial_{2,3} \\
-2 \cdot \partial_{2,2}-e_{1} \cdot \partial_{2,3}
\end{array}\right] .
$$


So

$$
\left[\begin{array}{l}
\partial_{1,1} \Theta \\
\partial_{1,2} \Theta
\end{array}\right]=\left[\begin{array}{cccc}
1 & e_{1} & 2 e_{2} & 0 \\
0 & -2 & -e_{1} & 0
\end{array}\right]\left[\begin{array}{l}
\partial_{1,1} \\
\partial_{2,2} \\
\partial_{2,3} \\
\partial_{2,4}
\end{array}\right]
$$

So

$$
\tilde{\mathrm{N}}=\left[\begin{array}{cccc}
1 & e_{1} & 2 e_{2} & 0 \\
0 & -2 & -e_{1} & 0
\end{array}\right]
$$

We may now express each of the $D^{m} \Theta^{i}$ in terms of the partials comprising $\mathcal{A}_{\Omega}$. We will express all monomials in the order $e_{1} \prec e_{2} \prec e_{1}^{\prime} \prec e_{2}^{\prime} \prec e_{1}^{\prime \prime} \prec e_{2}^{\prime \prime}$ :

$$
\begin{aligned}
\Theta & =e_{1} \cdot \partial_{1,1}-2 e_{2} \cdot \partial_{2,2}-2 e_{1} e_{2} \cdot \partial_{2,3}-2 e_{2}^{2} \cdot \partial_{2,4}, \\
D & =e_{1}^{\prime} \cdot \partial_{1,1}-e_{2}^{\prime} \cdot \partial_{2,2}-e_{2}^{\prime} e_{1} \cdot \partial_{2,3}-e_{2}^{\prime} e_{2} \cdot \partial_{2,4}, \\
D^{2} & =\left(e_{1}^{\prime \prime} \frac{\partial}{\partial e_{1}^{\prime}}+e_{2}^{\prime \prime} \frac{\partial}{\partial e_{2}^{\prime}}+e_{1}^{\prime} \frac{\partial}{\partial e_{1}}+e_{2}^{\prime} \frac{\partial}{\partial e_{2}}\right)\left(e_{1}^{\prime} \frac{\partial}{\partial e_{1}}+e_{2}^{\prime} \frac{\partial}{\partial e_{2}}\right),
\end{aligned}
$$

which implies

$$
D^{2}=e_{1}^{\prime \prime} \frac{\partial}{\partial e_{1}}+e_{2}^{\prime \prime} \frac{\partial}{\partial e_{2}}+\left(e_{1}^{\prime}\right)^{2} \frac{\partial^{2}}{\partial e_{1}^{2}}+2\left(e_{1}^{\prime} e_{2}^{\prime}\right) \frac{\partial^{2}}{\partial e_{1} \partial e_{2}}+\left(e_{2}^{\prime}\right)^{2} \frac{\partial^{2}}{\partial e_{2}^{2}}
$$

or

$$
D^{2}=e_{1}^{\prime \prime} \partial_{1,1}+e_{2}^{\prime \prime} \partial_{1,2}+\left(e_{1}^{\prime}\right)^{2} \partial_{2,2}+2\left(e_{1}^{\prime} e_{2}^{\prime}\right) \partial_{2,3}+\left(e_{2}^{\prime}\right)^{2} \partial_{2,4}
$$

So

$$
D^{2}=e_{1}^{\prime \prime} \partial_{1,1}-e_{2}^{\prime \prime}\left(\partial_{2,2}+e_{1} \partial_{2,3}+e_{2} \partial_{2,4}\right)+\left(e_{1}^{\prime}\right)^{2} \partial_{2,2}+2\left(e_{1}^{\prime} e_{2}^{\prime}\right) \partial_{2,3}+\left(e_{2}^{\prime}\right)^{2} \partial_{2,4}
$$

or

$$
\begin{gathered}
D^{2}=e_{1}^{\prime \prime} \partial_{1,1}+\left(\left(e_{1}^{\prime}\right)^{2}-e_{2}^{\prime \prime}\right) \cdot \partial_{2,2}+\left(2 e_{1}^{\prime} e_{2}^{\prime}-e_{1} e_{2}^{\prime \prime}\right) \cdot \partial_{2,3}+\left(\left(e_{2}^{\prime}\right)^{2}-e_{2} e_{2}^{\prime \prime}\right) \cdot \partial_{2,4} \cdot \\
D \Theta=\left(e_{1}^{\prime}\left(\partial / \partial e_{1}\right)+e_{2}^{\prime}\left(\partial / \partial e_{2}\right)\right)\left(e_{1}\left(\partial / \partial e_{1}\right)+2 e_{2}\left(\partial / \partial e_{2}\right)\right) \text {, which implies } \\
D \Theta=e_{1}^{\prime} \frac{\partial}{\partial e_{1}}+2 e_{2}^{\prime} \frac{\partial}{\partial e_{2}}+e_{1} e_{1}^{\prime} \frac{\partial^{2}}{\partial e_{1}^{2}}+\left(2 e_{2} e_{1}^{\prime}+e_{1} e_{2}^{\prime}\right) \frac{\partial^{2}}{\partial e_{1} \partial e_{2}}+2 e_{2} e_{2}^{\prime} \frac{\partial^{2}}{\partial e_{2}^{2}}
\end{gathered}
$$

or

$$
D \Theta=e_{1}^{\prime} \partial_{1,1}+2 e_{2}^{\prime} \partial_{1,2}+e_{1} e_{1}^{\prime} \partial_{2,2}+\left(2 e_{2} e_{1}^{\prime}+e_{1} e_{2}^{\prime}\right) \cdot \partial_{2,3}+2 e_{2} e_{2}^{\prime} \partial_{2,4},
$$

or, after using (10.2) to replace $\partial_{1,2}$,

$$
D \Theta=e_{1}^{\prime} \partial_{1,1}-2 e_{2}^{\prime}\left(\partial_{2,2}+e_{1} \partial_{2,3}+e_{2} \partial_{2,4}\right)+e_{1} e_{1}^{\prime} \partial_{2,2}+\left(2 e_{2} e_{1}^{\prime}+e_{1} e_{2}^{\prime}\right) \cdot \partial_{2,3}+2 e_{2} e_{2}^{\prime} \partial_{2,4}
$$


or

$$
D \Theta=e_{1}^{\prime} \partial_{1,1}+\left(e_{1} e_{1}^{\prime}-2 e_{2}^{\prime}\right) \cdot \partial_{2,2}+\left(2 e_{2} e_{1}^{\prime}-e_{1} e_{2}^{\prime}\right) \cdot \partial_{2,3}+0 \cdot \partial_{2,4}
$$

$\Theta^{2}=\left(e_{1} \cdot \partial_{1,1}+2 e_{2} \cdot \partial_{1,2}\right)\left(e_{1} \cdot \partial_{1,1}+2 e_{2} \cdot \partial_{1,2}\right)$, which implies

$$
\Theta^{2}=e_{1} \cdot \partial_{1,1}+4 e_{2} \cdot \partial_{1,2}+e_{1}^{2} \cdot \partial_{2,2}+4 e_{1} e_{2} \cdot \partial_{2,3}+4 e_{2}^{2} \cdot \partial_{2,4}
$$

So

$$
\Theta^{2}=e_{1} \cdot \partial_{1,1}-4 e_{2} \cdot\left(\partial_{2,2}+e_{1} \partial_{2,3}+e_{2} \partial_{2,4}\right)+e_{1}^{2} \cdot \partial_{2,2}+4 e_{1} e_{2} \cdot \partial_{2,3}+4 e_{2}^{2} \cdot \partial_{2,4}
$$

or

$$
\Theta^{2}=e_{1} \cdot \partial_{1,1}+\left(e_{1}^{2}-4 e_{2}\right) \cdot \partial_{2,2}+0 \cdot \partial_{2,3}+0 \cdot \partial_{2,4}
$$

We may summarize these partial derivative relations by

$$
\aleph_{\Omega} \equiv\left[\begin{array}{c}
D^{2} \\
D \\
D \Theta \\
\Theta \\
\Theta^{2}
\end{array}\right]=\left[\begin{array}{cccc}
e_{1}^{\prime \prime} & \left(e_{1}^{\prime}\right)^{2}-e_{2}^{\prime \prime} & 2 e_{1}^{\prime} e_{2}^{\prime}-e_{1} e_{2}^{\prime \prime} & \left(e_{2}^{\prime}\right)^{2}-e_{2} e_{2}^{\prime \prime} \\
e_{1}^{\prime} & -e_{2}^{\prime} & -e_{1} e_{2}^{\prime} & -e_{2} e_{2}^{\prime} \\
e_{1}^{\prime} & e_{1} e_{1}^{\prime}-2 e_{2}^{\prime} & 2 e_{2} e_{1}^{\prime}-e_{1} e_{2}^{\prime} & 0 \\
e_{1} & -2 e_{2} & -2 e_{1} e_{2} & -2 e_{2}^{2} \\
e_{1} & e_{1}^{2}-4 e_{2} & 0 & 0
\end{array}\right] \cdot\left[\begin{array}{c}
\partial_{1,1} \\
\partial_{2,2} \\
\partial_{2,3} \\
\partial_{2,4}
\end{array}\right]=\Sigma \cdot \mathcal{H}_{\Omega}
$$

where $\Sigma$ is defined as the $\Phi \times \Psi=5 \times 4$ transition matrix $\left[\tau_{(i, m) \times \kappa}\right]_{\substack{(i, m) \in S \\ \kappa \in[\Psi]}}$ from the vector of partial derivatives $\mathfrak{A}_{\Omega}$ to the vector of total derivatives $\aleph_{\Omega}$ as defined in Theorem 8.1. (We defined $\Sigma$ here in the application to a quadratic since we do not really need it elsewhere.)

11. Factoring the quadratic resolvent by Theorem 8.2. If a matrix $A$ is an odd permutation of the rows of a matrix $B$, then write $A \approx-B$. If a matrix $A$ is an even permutation of the rows of a matrix $B$, then write $A \approx B$. If $A \approx-B$, then $\operatorname{det}(A)=-\operatorname{det}(B)$. If $A \approx B$, then $\operatorname{det}(A)=\operatorname{det}(B)$. Then we have $A_{0,2}^{\dagger} \approx-\mathrm{A}_{0,2}=-\Lambda_{0,2} * \vec{p}$, where

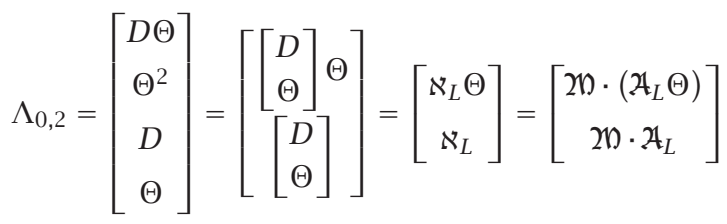

$$
\begin{aligned}
& =\left[\begin{array}{c}
2 \mathfrak{W} \cdot\left(\tilde{\mathrm{N}} * \mathfrak{A}_{\Omega}\right) \\
2 \mathfrak{W} \cdot\left(\mathrm{N} * \mathfrak{H}_{\Omega}\right)
\end{array}\right]=\left[\begin{array}{cc}
2 \mathfrak{W} & 0 \\
0 & 2 \mathfrak{W}
\end{array}\right] *\left[\begin{array}{c}
\tilde{\mathrm{N}} \\
\mathrm{N}
\end{array}\right] * \mathcal{H}_{\Omega} .
\end{aligned}
$$


We have just shown that $\operatorname{det} 2 \mathfrak{W}$ factors out of $\operatorname{det} A_{0,2}$ twice. $A_{0,1}^{\dagger} \approx \Lambda_{0,1} * \vec{p}$, where

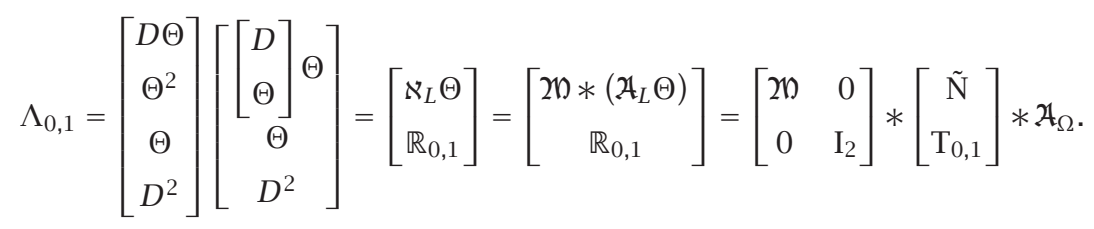

$A_{1,1}^{\dagger} \approx-\Lambda_{1,1} * \vec{p}$, where

$$
\Lambda_{1,1}=\left[\begin{array}{c}
D \\
\Theta \\
\Theta^{2} \\
D^{2}
\end{array}\right]=\left[\begin{array}{c}
\aleph_{L} \\
\mathbb{R}_{1,1}
\end{array}\right]=\left[\begin{array}{c}
2 \mathfrak{W} * \mathcal{A}_{L} \\
\mathbb{R}_{1,1}
\end{array}\right]=\left[\begin{array}{cc}
2 \mathfrak{W} & 0 \\
0 & \mathrm{I}_{2}
\end{array}\right] *\left[\begin{array}{c}
\mathrm{N} \\
\mathrm{T}_{1,1}
\end{array}\right] * \mathfrak{A}_{\Omega}
$$

$A_{1,0}^{\dagger} \approx \Lambda_{1,0} * \vec{p}$, where

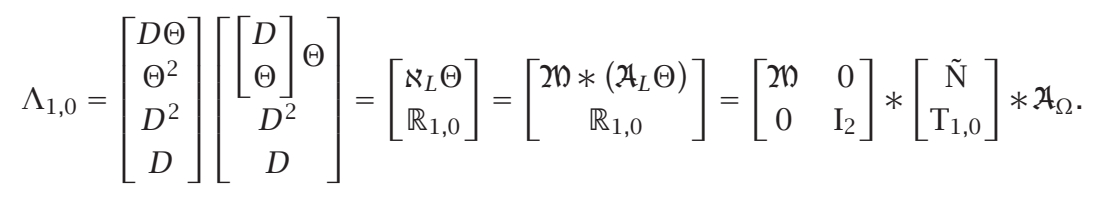

$A_{2,0}^{\dagger} \approx-\Lambda_{2,0} * \vec{p}$, where

$$
\Lambda_{2,0}=\left[\begin{array}{c}
D \\
\Theta \\
D^{2} \\
D \Theta
\end{array}\right]=\left[\begin{array}{c}
\mathfrak{\aleph}_{L} \\
\mathbb{R}_{2,0}
\end{array}\right]=\left[\begin{array}{c}
2 \mathfrak{W} * \mathfrak{A}_{L} \\
\mathbb{R}_{2,0}
\end{array}\right]=\left[\begin{array}{cc}
2 \mathfrak{W} & 0 \\
0 & \mathrm{I}_{2}
\end{array}\right] *\left[\begin{array}{c}
\mathrm{N} \\
\mathrm{T}_{2,0}
\end{array}\right] * \mathcal{H}_{\Omega} .
$$

We remark that $\mathrm{T}_{0,1}$ is the 4 th and 1 st rows of $\Sigma, \mathrm{T}_{1,1}$ is the 5 th and 1 st rows of $\Sigma, \mathrm{T}_{1,0}$ is the 1 st and 2 nd rows of $\Sigma$, and $T_{2,0}$ is the 1 st and 3rd rows of $\Sigma$. Thus

$$
\begin{aligned}
& F_{0,2}=-\operatorname{det}\left(\Lambda_{0,2} * \vec{p}\right)=-(\operatorname{det} 2 \mathfrak{W})^{2} \cdot \operatorname{det}\left[\begin{array}{c}
\tilde{\mathrm{N}} \\
\mathrm{N}
\end{array}\right] \cdot \operatorname{det}\left(\mathcal{A}_{\Omega} * \vec{p}\right), \\
& F_{0,1}=-\operatorname{det}\left(\Lambda_{0,1} * \vec{p}\right)=-(\operatorname{det} 2 \mathfrak{W}) \cdot \operatorname{det}\left[\begin{array}{c}
\tilde{\mathrm{N}} \\
\mathrm{T}_{0,1}
\end{array}\right] \cdot \operatorname{det}\left(\mathcal{A}_{\Omega} * \vec{p}\right), \\
& F_{1,1}=-\operatorname{det}\left(\Lambda_{1,1} * \vec{p}\right)=-(\operatorname{det} 2 \mathfrak{W}) \cdot \operatorname{det}\left[\begin{array}{c}
\mathrm{N} \\
\mathrm{T}_{1,1}
\end{array}\right] \cdot \operatorname{det}\left(\mathcal{A}_{\Omega} * \vec{p}\right), \\
& F_{1,0}=-\operatorname{det}\left(\Lambda_{1,0} * \vec{p}\right)=-(\operatorname{det} 2 \mathfrak{W}) \cdot \operatorname{det}\left[\begin{array}{c}
\tilde{\mathrm{N}} \\
\mathrm{T}_{1,0}
\end{array}\right] \cdot \operatorname{det}\left(\mathfrak{A}_{\Omega} * \vec{p}\right), \\
& F_{2,0}=-\operatorname{det}\left(\Lambda_{2,0} * \vec{p}\right)=-(\operatorname{det} 2 \mathfrak{W}) \cdot \operatorname{det}\left[\begin{array}{c}
\mathrm{N} \\
\mathrm{T}_{2,0}
\end{array}\right] \cdot \operatorname{det}\left(\mathfrak{A}_{\Omega} * \vec{p}\right) .
\end{aligned}
$$

Division of each coefficient function $F_{i, m}$ by $\operatorname{det}(\mathfrak{W}) \cdot \operatorname{det}\left(\mathcal{A}_{\Omega} * \vec{p}\right)$ yields $\theta_{i, m}$, the coefficient functions of the Cohnian of $P$. We note that $\mathfrak{A}_{\Omega} * \vec{p}$ is a $4 \times 4$ upper-triangular matrix whose main diagonal equals $\left[\begin{array}{llll}\partial_{1,1} p_{1} & \partial_{2,2} p_{2} & \partial_{2,3} p_{3} & \partial_{2,4} p_{4}\end{array}\right]=\left[\begin{array}{llll}1 & 2 & -3 & 4\end{array}\right]$. 
Therefore $\operatorname{det}\left(\mathcal{A}_{\Omega} * \vec{p}\right)=-24$.

$$
\begin{aligned}
& \theta_{0,2}=-(\operatorname{det} 2 \mathfrak{h}) \cdot \operatorname{det}\left[\begin{array}{c}
\tilde{\mathrm{N}} \\
\mathrm{N}
\end{array}\right]=-\left|\begin{array}{cc}
e_{1}^{\prime} & e_{2}^{\prime} \\
1 \cdot e_{1} & 2 \cdot e_{2}
\end{array}\right| \cdot\left|\begin{array}{cccc}
1 & 0 & 0 & 0 \\
0 & -1 & -e_{1} & -e_{2} \\
1 & e_{1} & 2 e_{2} & 0 \\
0 & -2 & -e_{1} & 0
\end{array}\right| \\
& =\left|\begin{array}{cc}
1 \cdot e_{1} & 2 \cdot e_{2} \\
e_{1}^{\prime} & e_{2}^{\prime}
\end{array}\right| \cdot e_{2} \cdot \Delta, \\
& \theta_{0,1}=-\operatorname{det}\left[\begin{array}{c}
\tilde{\mathrm{N}} \\
\mathrm{T}_{0,1}
\end{array}\right]=-\left|\begin{array}{cccc}
1 & e_{1} & 2 e_{2} & 0 \\
0 & -2 & -e_{1} & 0 \\
e_{1} & -2 e_{2} & -2 e_{1} e_{2} & -2 e_{2}^{2} \\
e_{1}^{\prime \prime} & \left(e_{1}^{\prime}\right)^{2}-e_{2}^{\prime \prime} & 2 e_{1}^{\prime} e_{2}^{\prime}-e_{1} e_{2}^{\prime \prime} & \left(e_{2}^{\prime}\right)^{2}-e_{2} e_{2}^{\prime \prime}
\end{array}\right| \text {, } \\
& \theta_{1,1}=-\operatorname{det}\left[\begin{array}{c}
\mathrm{N} \\
\mathrm{T}_{1,1}
\end{array}\right]=-\left|\begin{array}{cccc}
1 & 0 & 0 & 0 \\
0 & -1 & -e_{1} & -e_{2} \\
e_{1} & e_{1}^{2}-4 e_{2} & 0 & 0 \\
e_{1}^{\prime \prime} & \left(e_{1}^{\prime}\right)^{2}-e_{2}^{\prime \prime} & 2 e_{1}^{\prime} e_{2}^{\prime}-e_{1} e_{2}^{\prime \prime} & \left(e_{2}^{\prime}\right)^{2}-e_{2} e_{2}^{\prime \prime}
\end{array}\right| \text {, } \\
& \theta_{1,0}=-\operatorname{det}\left[\begin{array}{c}
\tilde{\mathrm{N}} \\
\mathrm{T}_{1,0}
\end{array}\right]=-\left|\begin{array}{cccc}
1 & e_{1} & 2 e_{2} & 0 \\
0 & -2 & -e_{1} & 0 \\
e_{1}^{\prime \prime} & \left(e_{1}^{\prime}\right)^{2}-e_{2}^{\prime \prime} & 2 e_{1}^{\prime} e_{2}^{\prime}-e_{1} e_{2}^{\prime \prime} & \left(e_{2}^{\prime}\right)^{2}-e_{2} e_{2}^{\prime \prime} \\
e_{1}^{\prime} & -e_{2}^{\prime} & -e_{1} e_{2}^{\prime} & -e_{2} e_{2}^{\prime}
\end{array}\right| \text {, } \\
& \theta_{2,0}=-\operatorname{det}\left[\begin{array}{c}
\mathrm{N} \\
\mathrm{T}_{2,0}
\end{array}\right]=-\left|\begin{array}{cccc}
1 & 0 & 0 & 0 \\
0 & -1 & -e_{1} & -e_{2} \\
e_{1}^{\prime \prime} & \left(e_{1}^{\prime}\right)^{2}-e_{2}^{\prime \prime} & 2 e_{1}^{\prime} e_{2}^{\prime}-e_{1} e_{2}^{\prime \prime} & \left(e_{2}^{\prime}\right)^{2}-e_{2} e_{2}^{\prime \prime} \\
e_{1}^{\prime} & e_{1} e_{1}^{\prime}-2 e_{2}^{\prime} & 2 e_{1}^{\prime} e_{2}-e_{1} e_{2}^{\prime} & 0
\end{array}\right| .
\end{aligned}
$$

The weight of each $F_{i, m}$ is $\Psi \cdot(\Psi+1) / 2=10$. We factored out $24 \cdot \operatorname{det} 2 \mathrm{~b}$ to obtain $\theta_{i, m}$ which has weight 7 . One can divide the Cohnian by the leading term to get a nonintegral monic resolvent, $D^{2} y+\left(g_{0,1}+g_{1,1} \cdot \alpha\right) \cdot D y+\left(g_{1,0} \cdot \alpha+g_{2,0} \cdot \alpha^{2}\right) \cdot y=0$. One can show that

$$
\begin{gathered}
g_{0,1}=-\frac{D W}{W}+\frac{1}{2} \frac{D \Delta}{\Delta}+\frac{D e_{2}}{e_{2}}, \\
g_{1,1}=-\frac{D e_{2}}{e_{2}} \\
g_{1,0}=\frac{1}{2}\left(\frac{D e_{2}}{e_{2}} \cdot\left(\frac{D W}{W}-\frac{1}{2} \frac{D \Delta}{\Delta}\right)-\frac{D^{2} e_{2}}{e_{2}}\right), \\
g_{2,0}=\frac{1}{2}\left(\frac{D W}{W} \cdot \frac{D \Delta}{\Delta}-\frac{D^{2} \Delta}{\Delta}\right)-g_{1,0},
\end{gathered}
$$

where $W \equiv\left|\begin{array}{cc}1 \cdot e_{1} & 2 \cdot e_{2} \\ e_{1}^{\prime} & e_{2}^{\prime}\end{array}\right|=-\operatorname{det} 2 \mathfrak{W}$ is $\sqrt{\Delta} / \alpha \cdot e_{2}^{\alpha-1}$ times the Wronskian.

12. Factoring a cubic by Mathematica. We will now present an example which requires the use of a computer algebra system to test the conjecture that the determinant of the matrix $2 \mathfrak{W}$ given by (8.5) in Section 8 divides every term of the resolvent obtained 
by the powersum formula (3.1). Consider the cubic (3.1) in $z$

$$
z^{3}-x \cdot z^{2}+x^{2} \cdot z-\left(3 x^{3}+2 x^{2}-11 x-10\right)=0
$$

with $D x=1$. The form of the third-order $\alpha$-resolvent of a general cubic was given by (4.5) where $n=3, \Omega=4$ by (1.1), and $L=3$. Let $g(x)$ denote the greatest common divisor in the ring $\mathbb{Z}[x]$ of all thirteen $F_{i, m}$ in this resolvent.

Let $2 \mathfrak{W}$ denote the $9 \times 9$ transition matrix from the $9 \times 1$ column vector of partial differential operators $\mathcal{A}_{L} \equiv\left[\partial_{1,1}, \partial_{2,2}, \partial_{3,3}, \partial_{3,4}, \partial_{3,5}, \partial_{3,6}, \partial_{3,7}, \partial_{3,8}, \partial_{3,9}\right]^{\dagger}$ to the $9 \times 1$ column vector of operators $\kappa_{L} \equiv\left[D, \Theta, D^{2}, D \Theta, \Theta^{2}, D^{3}, D^{2} \Theta, D \Theta^{2}, \Theta^{3}\right]^{\dagger}$. One first computes the entries of $2 \mathfrak{W}$ as elements of $\mathbb{Q}\left\{e_{1}, e_{2}, e_{3}\right\}$ and then specializes $e_{1} \rightarrow x, e_{2} \rightarrow x^{2}, e_{3} \rightarrow$ $\left(3 x^{3}+2 x^{2}-11 x-10\right), e_{1}^{\prime} \rightarrow 1$, and so forth. Since $\kappa_{L}=2 \mathfrak{W} * \mathcal{L}_{L}$, we may apply this $9 \times 1$ vector to the $1 \times 9$ row vector of the first nine powersums $\vec{p}$ to get the $9 \times 9$ matrix $\overline{2 \mathfrak{W}} \equiv \kappa_{L} \vec{p}=\mathfrak{W} *\left(\mathfrak{A}_{L} \vec{p}\right)$. Since $\mathfrak{A}_{L} \vec{p}$ is a $9 \times 9$ upper-triangular matrix whose main diagonal is $[1,2,6,-8,10,-12,14,-16,18]$ we have that $\operatorname{det}(\overline{2 \mathfrak{W}})=-46448640$. $\operatorname{det}(\mathfrak{W})=2^{14} \cdot 3^{4} \cdot 5^{1} \cdot 7^{1} \cdot \operatorname{det}(\mathfrak{W})$. The computer directly computes $\overline{\mathfrak{W}}$, not $\mathfrak{2}$, since $\overline{2 \mathfrak{V}}=\left[D^{m^{\prime}} q^{i^{\prime}} p_{q}\right]_{\left(i^{\prime}, m^{\prime}\right) \times q, 1 \leq q \leq 9}$ is very easy to compute.

$$
D^{m^{\prime}} q^{i^{\prime}} \in \mathcal{N}_{3}
$$

The author used Mathematica 4.0 for Students on a Dell Dimension XPS R400 computer, Windows 98. The computation time was about 5 minutes. The computer found that

$$
\begin{aligned}
g(x)= & 2^{31} \cdot 3^{9} \cdot 5^{2} \cdot 7^{1} \cdot 11^{1} \cdot(-2+x) \cdot x^{2} \cdot(1+x) \cdot(5+3 x) \\
& \cdot\left(-15-11 x+x^{2}\right)^{11} \cdot \psi(x) \cdot \phi(x)
\end{aligned}
$$

where

$$
\phi(x) \equiv\left(2700+5940 x+2187 x^{2}-2688 x^{3}-1520 x^{4}+296 x^{5}+204 x^{6}\right),
$$

$\psi(x) \equiv\left(-10-11 x+2 x^{2}+6 x^{3}\right)$, and $\operatorname{det}(\overline{2 \mathfrak{W}})=2^{22} \cdot 3^{5} \cdot 5^{1} \cdot 7^{1} \cdot x^{2}\left(-15-11 x+x^{2}\right)^{10}$. $\psi(x)$. So $\operatorname{deg}_{x} g(x)=36$. One sees that $\operatorname{det}(\overline{2 \mathfrak{W}})$, and hence $\operatorname{det}(2 \mathfrak{W})$, divides $g$ in the ring $\mathbb{Q}[x]$. Therefore, the conjecture has been verified for $P(t) \equiv t^{3}-x \cdot t^{2}+x^{2} \cdot t-\left(3 x^{3}+\right.$ $\left.2 x^{2}-11 x-10\right)$.

We also note from the output of this program that the leading term in the resolvent $F_{0,3}+\alpha \cdot F_{1,3}$ equals

$$
\begin{aligned}
(-2+x) \cdot(1+x) \cdot(5+3 x) \cdot\left(-15-11 x+x^{2}\right)^{2} \\
\cdot \phi(x) \cdot\left(\psi(x)+\alpha\left(50+55 x-10 x^{2}-18 x^{3}\right)\right) .
\end{aligned}
$$

At $x \in\{2,-1,-5 / 3\}$ and the roots of $-15-11 x+x^{2}$ and $\phi(x)$, the powersum formula fails, since the formula yields an identically zero differential equation. However, for all powers, $\alpha \neq 1 / 5$, there exist roots of $\left(\psi(x)+\alpha \cdot\left(50+55 x-10 x^{2}-18 x^{3}\right)\right)$ which are not roots of $g(x)$. These yield the apparent singularities of the resolvent. At $\alpha=$ $1 / 5, F_{0,3}+\alpha \cdot F_{1,3}$ reduces to $(12 / 5) x^{3}$ whose only root is $x=0$, which satisfies $g(0)=0$. 
Appendix. We now state two theorems regarding the existence and form of resolvents for any polynomial with differentiable coefficients. We will prove in a nonrigorous manner the main result of the second theorem asserting the maximal degree of $\alpha$.

THEOREM A.1. Let $P(t) \equiv \sum_{i=0}^{N}(-1)^{N-i} e_{N-i} \cdot t^{i}$ be an Nth-degree polynomial over a $d$-field, $\mathbb{F}$, with $n$ distinct roots $\left\{z_{j}\right\}_{j=1}^{n}$. Let $\alpha$ be either a transcendental indeterminate over $\mathbb{F}$ with $D \alpha=0$ or an integer. For each $j \in[n]$, let $y_{j}$ be a nonzero solution to the ordinary differential equation, $z_{j} \cdot D y_{j}=\alpha \cdot y_{j} \cdot D z_{j}$. Define o to be the maximal number of the $y \equiv\left\{y_{j}\right\}_{j=1}^{n}$ which are linearly independent over constants. Then there exists a nonzero oth-order $\alpha$-resolvent, $\mathfrak{k}$, of $P$ with terms in $\mathbb{Z}\{e\}_{o}[\alpha]$. Furthermore, no $\alpha$-resolvent of $P$ has order less than $o$.

REMARKS A.2. This is [6, Theorem 16, page 39]. Theorem A.1 asserts a little more than the well-known result that there exists a linear ordinary differential equation of order $o$ which is satisfied by any set of $o$ functions which are linearly independent over constants. One has to work through the details that the terms of this linear ordinary differential equation lie in the desired differential ring.

THEOREM A.3. Let $P(t) \equiv \sum_{i=0}^{N}(-1)^{n-i} e_{N-i} \cdot t^{i} \in \mathbb{F}[t]$ be a polynomial of degree $N$ in $t$ over a d-field, $\mathbb{F}$, with $n$ distinct roots $\left\{z_{i}\right\}_{i=1}^{n}$. Then there exists an oth-order differential resolvent, $\wp y \equiv \sum_{m=0}^{o} \sum_{i=0}^{\Omega-m} R_{i, m} \cdot \alpha^{i} D^{m} y=0$, with $R_{i, m} \in \mathbb{Z}\{e\}_{o}$ and $R_{0,0}=0$ for some $o \in[n]$ where $\Omega$ is defined to be $o(o-1) / 2+1$. Furthermore, o may be chosen to equal the number of $\left\{y_{j}\right\}_{j=1}^{n}$ linearly independent over constants.

Proof. If $P(t) \equiv t^{v} \cdot \bar{P}(t)$, where $\bar{P}(t)$ has no zero roots, then $\bar{P}$ and $P$ have the same differential coefficient field $\mathbb{Q}\langle e\rangle$ and the same nonzero roots. The zero roots of $\bar{P}$ satisfy the resolvent of $P$, since a resolvent is a homogeneous linear ordinary differential equation. So $\bar{P}$ and $P$ have the same resolvent. So, henceforth, assume $v=0$. Write $P(t)=\prod_{i=1}^{n}\left(t-z_{i}\right)^{\pi_{t}}$.

Define

$$
\begin{aligned}
& b_{k} \equiv(-1)^{m} \frac{\left|D p_{m} / m \quad p_{m+i}\right|_{\substack{m \times i, 1 \leq m \leq n \\
i \neq k, 0 \leq i \leq n-1}}}{\left|p_{m+i}\right|_{\substack{m \times i, 1 \leq m \leq n \\
0 \leq i \leq n-1}}} \\
& =(-1)^{m} \frac{\left|\sum_{j=1}^{n} \pi_{j} \cdot z_{j}^{m-1} D z_{j} \quad \sum_{j=1}^{n} \pi_{j} \cdot z_{j}^{m+i}\right|_{\substack{m \times i, 1 \leq m \leq n \\
i \neq k, 0 \leq i \leq n-1}}}{\left|\sum_{j=1}^{n} \pi_{j} \cdot z_{j}^{m+i}\right|_{\substack{m \times i, 1 \leq m \leq n \\
0 \leq i \leq n-1}}} .
\end{aligned}
$$

By definition, $b_{k} \in \mathbb{Q}\langle e\rangle$. Factoring the denominator and numerator yields

$$
\begin{aligned}
b_{k} & =(-1)^{m} \frac{\left|\pi_{j} \cdot z_{j}^{m}\right|_{m \times j} \cdot\left|z_{j}^{-1} \cdot D z_{j} \quad z_{j}^{i}\right|_{j \times i, i \neq k, 0 \leq i \leq n-1}}{\left|\pi_{j} \cdot z_{j}^{m}\right|_{m \times j} \cdot\left|z_{j}^{i}\right|_{j \times i, 0 \leq i \leq n-1}} \\
& =(-1)^{m} \frac{\left|z_{j}^{-1} \cdot D z_{j} \quad z_{j}^{i}\right|_{i \times j, i \neq k, 0 \leq i \leq n-1}}{\left|z_{j}^{i}\right|_{i \times j, 0 \leq i \leq n-1}} .
\end{aligned}
$$


Thus $b_{k}$ is simply the Cramer's rule solution of the system of $n$ linear equations $D z_{j} / z_{j}=\sum_{k=0}^{n-1} b_{k} \cdot z_{j}^{k}$ for each $j \in[n]$.

For any root $z$, define $B_{11}(z) \equiv \sum_{k=0}^{n-1} b_{k} \cdot z^{k}$. Then $D z^{\alpha}=\alpha \cdot z^{\alpha} \cdot(D z / z)=\alpha \cdot z^{\alpha}$. $B_{11}(z)$. So the hypothesis that $D^{m} z^{\alpha} / z^{\alpha}$ is a polynomial in $\alpha$ over $\mathbb{Q}\langle e\rangle[z]$ of degree at most $m$ is true for $m=1$. Assume that $D^{m} z^{\alpha}=z^{\alpha} \cdot \sum_{l=1}^{m} \alpha^{l} \cdot B_{l, m}(z)$, where $B_{l, m}(z) \in \mathbb{Q}\langle e\rangle[z]$. Then $D^{m+1} z^{\alpha}=\left(D z^{\alpha}\right) \cdot \sum_{l=1}^{m} \alpha^{l} \cdot B_{l, m}(z)+z^{\alpha} \cdot \sum_{l=1}^{m} \alpha^{l} \cdot D B_{l, m}(z)=\alpha$. $z^{\alpha} B_{1,1}(z) \cdot \sum_{l=1}^{m} \alpha^{l} \cdot B_{l, m}(z)+z^{\alpha} \cdot \sum_{l=1}^{m} \alpha^{l} \cdot D B_{l, m}(z)=z^{\alpha} \cdot \sum_{l=1}^{m+1} \alpha^{l} \cdot\left(B_{1,1}(z) \cdot B_{l-1, m}(z)+\right.$ $D B_{l, m}(z)$ ), where $B_{0, m}(z) \equiv 0$ for $m \in[n]$ and $B_{m+1, m}(z) \equiv 0$. So the hypothesis is true for all $m$.

Define $B_{0,0}(z) \equiv 1$. Let $o$ denote the maximal number of $\left\{y_{i}\right\}_{i=1}^{n}=\left\{z_{i}^{\alpha}\right\}_{i=1}^{n}$ which are linearly independent over constants. Let $\left\{y_{i}\right\}_{i=1}^{o}=\left\{z_{i}^{\alpha}\right\}_{i=1}^{o}$ be linearly independent over constants. Each of the $\left\{z_{i}^{\alpha}\right\}_{i=1}^{o}$ clearly satisfies the linear ordinary differential equation

$$
\begin{aligned}
& \mathfrak{J} y \equiv\left|D^{m} z_{i}^{\alpha} \quad D^{m} y\right|_{\substack{i \times m, 1 \leq i \leq o \\
0 \leq m \leq o}} \\
& =\left(\prod_{i=1}^{o} z_{i}^{\alpha}\right) \cdot \sum_{m=0}^{o}\left|\sum_{l=0}^{m^{\prime}} \alpha^{l} \cdot B_{l, m^{\prime}}\left(z_{i}\right)\right|_{\substack{i \times m^{\prime} \\
m^{\prime} \neq m, 0 \leq m^{\prime} \leq o}} \cdot D^{m} y=0 .
\end{aligned}
$$

The remaining $\left\{z_{i}^{\alpha}\right\}_{i=o+1}^{n}$ also satisfy this linear ordinary differential equation because they are linear combinations of $\left\{z_{i}^{\alpha}\right\}_{i=1}^{o}$ over constants. The leading term of $\mathfrak{J}$ is

$$
\begin{aligned}
W & \left.\equiv\left|D^{m} z_{i}^{\alpha}\right|_{\substack{i \times m, 1 \leq i \leq o \\
0 \leq m \leq 0-1}}^{o}{ }^{o} \prod_{i=1}^{\alpha} z_{i}^{\alpha}\right) \cdot\left|\sum_{l=0}^{o-1} \alpha^{l} \cdot B_{l, m^{\prime}}\left(z_{i}\right)\right|_{\substack{i \times m^{\prime}, 1 \leq i \leq 0 \\
0 \leq m^{\prime} \leq 0-1}},
\end{aligned}
$$

the Wronskian of $\mathfrak{J}$. Since the $\left\{z_{i}^{\alpha}\right\}_{i=1}^{o}$ are linearly independent over constants, $W \neq 0$. Let

$$
R_{m} \equiv \frac{\left|\sum_{l=0}^{m^{\prime}} \alpha^{l} \cdot B_{l, m^{\prime}}\left(z_{i}\right)\right|_{\substack{i \times m^{\prime} \\ m^{\prime} \neq m, 0 \leq m^{\prime} \leq o}}}{\left|\sum_{l=0}^{o-1} \alpha^{l} \cdot B_{l, m^{\prime}}\left(z_{i}\right)\right|_{\substack{i \times m^{\prime}, 1 \leq i \leq o \\ 0 \leq m^{\prime} \leq o-1}}} .
$$

Then $\mathfrak{J} y / W=D^{o} y+\sum_{m=0}^{o-1} R_{m} \cdot D^{m} y$ and $R_{m} \in \mathbb{Q}\langle e\rangle\left(z_{1}, \ldots, z_{o}, \alpha\right)$. Since $R_{m}$ no longer involves the $\alpha$ th power of $z$, we may apply each automorphism $\sigma$ in the Galois group $G$ of $P$ to this equation and sum the resulting equations to get $\hat{x} y \equiv \sum_{\sigma \in G} \sigma(\mathfrak{J} / W) y=$ $|G| \cdot D^{o} y+\sum_{m=0}^{o-1} \mathfrak{k}_{m} \cdot D^{m} y$, where the terms $\mathfrak{k}_{m}$ of $\hat{\mathfrak{z}}$ now lie in $\mathbb{Q}\langle e\rangle(\alpha)$ since they are now fixed under the action of any element in $G$. Since $|G| \neq 0, \hat{\mathfrak{R}}$ is not identically zero. So $\hat{\mathfrak{k}}$ is a resolvent of $P$. Clearing denominators of $\hat{\mathfrak{k}}$ yields an integral resolvent $\mathfrak{z}$ whose terms lie in $\mathbb{Z}\{e\}_{0}$. This proves Theorem A.1.

The resolvent $\hat{\imath}$ is a rational function of $\alpha$. Because we have summed over all elements in the Galois group to get $\hat{\varkappa}$, when we clear denominators to get $\mathfrak{\varkappa}$ we will get a resolvent which will be a polynomial in $\alpha$ of degree much greater than the result desired in Theorem A.3. Therefore, we must try a different approach which is quite similar to the standard proof of the existence of a resolvent for an irreducible polynomial which uses the existence of a basis for any vector space over $\mathbb{Q}\langle e\rangle(\alpha)$. 
Write $\sum_{l=0}^{m} \alpha^{l} \cdot B_{l, m}(z)=\sum_{k=0}^{n-1} z^{k} \cdot E_{m, k}$, where $E_{m, k} \in \mathbb{Q}\langle e\rangle[\alpha], E_{0, k}=1$, for all $k \in$

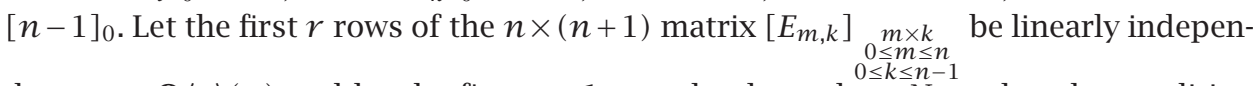
dent over $\mathbb{Q}\langle e\rangle(\alpha)$ and let the first $r+1$ rows be dependent. Note that the condition that $E_{0, k}=1$, for all $k \in[n-1]_{0}$, guarantees that $r>0$. So the $(r+1) \times(n+1)$ submatrix $\left[E_{m, k}\right] \underset{\substack{m \times m \leq r \\ 0 \leq k \leq n-1}}{\substack{0 \leq k \\ 0}}$ has rank $r$. Then this submatrix has an $r \times r$ submatrix $\mathrm{E}=\left[E_{m, k}\right]_{\substack{0 \leq m \leq r \\ k \in K}}$ for some subset $K \subset[n-1]_{0}$ such that $\operatorname{det} E \neq 0$. Hence,

$$
\begin{aligned}
\mathfrak{J} y & \equiv \sum_{m=0}^{r}\left|E_{m^{\prime}, k^{\prime}}\right|_{\substack{0 \leq m^{\prime} \leq r, m^{\prime} \neq m \\
k^{\prime} \in K}} \cdot(-1)^{m} \cdot D^{m} y \\
& =\sum_{m=0}^{r} \sum_{k=0}^{n-1} z^{k}\left|E_{m^{\prime}, k^{\prime}}\right|_{\substack{0 \leq m^{\prime} \leq r, m^{\prime} \neq m \\
k^{\prime} \in K}} \cdot(-1)^{m} \cdot E_{m, k} \\
& =\sum_{k=0}^{n-1} z^{k} \cdot \sum_{m=0}^{r}\left|E_{m^{\prime}, k^{\prime}}\right|_{\substack{0 \leq m^{\prime} \leq r, m^{\prime} \neq m \\
k^{\prime} \in K}} \cdot(-1)^{m} \cdot E_{m, k}=\sum_{k=0}^{n-1} z^{k} \cdot\left|E_{m, k^{\prime}}\right| \begin{array}{c}
\substack{m \times k^{\prime} \\
0 \leq m \leq k \\
k^{\prime} \in K \bigcup\{k\}} \\
k=0
\end{array}
\end{aligned}
$$

But the $(r+1) \times(r+1)$ determinant $\left|E_{m, k^{\prime}}\right|_{\substack{\begin{subarray}{c}{x \times k^{\prime}, 0 \leq m \leq r \\ k^{\prime} \in K \cup\{k\}} }} \\{\text { s. }}\end{subarray}}=0$ since $\left[E_{m, k}\right]_{\substack{m \times k, 0 \leq m \leq r \\ 0 \leq k \leq n-1}}$ has rank $r$. So $\mathfrak{J} y=0$. Since the leading term of $\mathfrak{J}$ is det $\mathbb{E} \neq 0$, and since $\left|E_{m^{\prime}, k^{\prime}}\right|_{\substack{0 \leq m^{\prime} \leq r, m^{\prime} \neq m \\ k^{\prime} \in K}} \in$ $\mathbb{Q}\langle e\rangle[\alpha], \mathfrak{J}$ is a resolvent.

Since $\operatorname{deg}_{\alpha} E_{m, k} \leq m$, it follows that $\operatorname{deg}_{\alpha}\left|E_{m^{\prime}, k^{\prime}}\right|_{\substack{0 \leq m^{\prime} \leq r, m^{\prime} \neq m \\ k^{\prime} \in K}} \leq \sum_{i=0}^{r} i-m=r \cdot(r+$ $1) / 2-m$. Since $B_{0, m^{\prime}}(z) \equiv 0$ for each $m^{\prime} \in[n], \alpha$ divides $E_{m^{\prime}, k^{\prime}}$ for each $m^{\prime} \in[n]$ and each $k^{\prime} \in K$. So, for each $m \in[r]$, we can factor $\alpha$ out of the $m^{\prime}$ th row of $\left|E_{m^{\prime}, k^{\prime}}\right|_{\substack{0 \leq m^{\prime} \leq r \\ m^{\prime} \neq m \\ k^{\prime} \in K}}$ for each $m^{\prime} \in[r], m^{\prime} \neq m$. Thus we can factor $\alpha^{r-1}$ out of $\left|E_{m^{\prime}, k^{\prime}}\right|_{0 \leq m^{\prime} \leq r, m^{\prime} \neq m}$. By a similar reasoning, we can factor $\alpha^{r}$ out of $\left|E_{m^{\prime}, k^{\prime}}\right|_{\substack{m^{\prime} \in[r] \\ k^{\prime} \in K}}$, the coefficient of $y$ in $\mathfrak{J} y$. Thus the resolvent $\wp \equiv \alpha^{-(r-1)} \cdot \mathfrak{J}$ has the form

$$
\wp y=\sum_{m=0}^{r} \sum_{i=0}^{\Omega-m} R_{i, m} \cdot \alpha^{i} D^{m} y,
$$

where $\Omega \equiv r \cdot(r+1) / 2-(r-1)=r \cdot(r-1) / 2+1$. This proves Theorem A.3.

REMARKS A.4. Theorem A.3 is [6, Theorem 37, page 67]. We used the letter $B$ to suggest base field. Referees at several other journals suggested using the left greatest common divisors of the differential resolvents of the irreducible factors of $P$ to prove Theorem A.3. However, even for an irreducible polynomial, one would still have to go through the steps in this proof to get the bounds on the degree in $\alpha$. A greater complication is proving that the resulting linear ordinary differential equation is in fact a resolvent of $P$. The resolvents of the irreducible factors of $P$ lie in the differential coefficient fields of the individual factors. But it is very difficult to prove that the terms of some combination of these resolvents lie in the differential coefficient field of the original polynomial $P$. 
ACKNOWLEDGMENT. The author acknowledges the great assistance of Dr. Richard Cohn, the author's doctoral dissertation adviser at Rutgers University. Dr. Michael Singer was instrumental in locating important, recent references for the author for formulae that were needed in this work and offered advice on how best to publish the appendix.

\section{REFERENCES}

[1] I. M. Gelfand, M. M. Kapranov, and A. V. Zelevinsky, Hypergeometric functions and toral manifolds, Funct. Anal. Appl. 23 (1989), no. 2, 94-106.

[2] R. Harley, On the theory of the transcendental solution of algebraic equations, Quarterly Journal of Pure and Applied Mathematics 5 (1862), 337-359.

[3] E. R. Kolchin, Differential Algebra and Algebraic Groups, Pure and Applied Mathematics, vol. 54, Academic Press, New York, 1973.

[4] I. G. Macdonald, Symmetric Functions and Hall Polynomials, 2nd ed., Oxford Mathematical Monographs, The Clarendon Press, Oxford University Press, New York, 1995.

[5] K. Mayr, Über die Lösung algebraischer Gleichungssysteme durch hypergeometrische Funktionen, Monatsh. Math. Phys. 45 (1937), 280-313 (German).

[6] J. M. Nahay, Linear differential resolvents, Doctoral dissertation, Rutgers University, New Jersey, 2000.

[7] _ Powersum formula for polynomials whose distinct roots are differentially independent over constants, Int. J. Math. Math. Sci. 32 (2002), no. 12, 721-738.

[8] _ Linear relations among algebraic solutions of differential equations, J. Differential Equations 191 (2003), no. 2, 323-347.

[9] _ Difference resolvents of minimal order and weight, Int. J. Math. Math. Sci. 2004 (2004), no. 54, 2867-2894.

[10] _ Powersum formula for differential resolvents, Int. J. Math. Math. Sci. 2004 (2004), no. 7, 365-371.

John Michael Nahay: Swan Orchestral Systems, 25 Chestnut Hill Lane, Columbus, NJ 080221039, USA

E-mail address: resolvent@comcast.net 


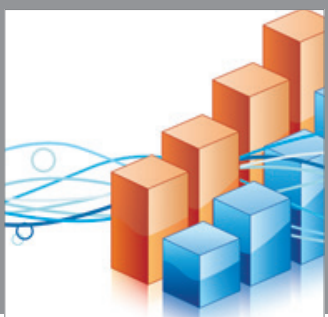

Advances in

Operations Research

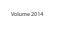

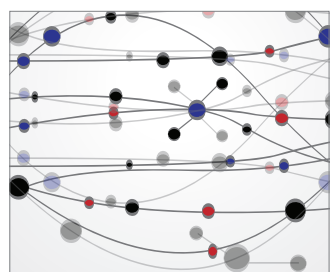

\section{The Scientific} World Journal
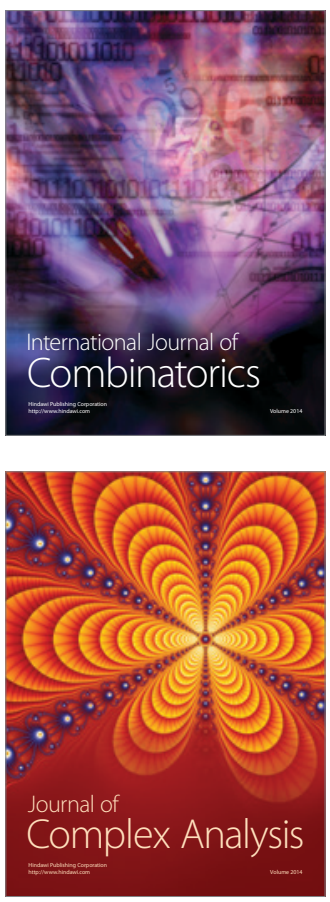

International Journal of

Mathematics and

Mathematical

Sciences
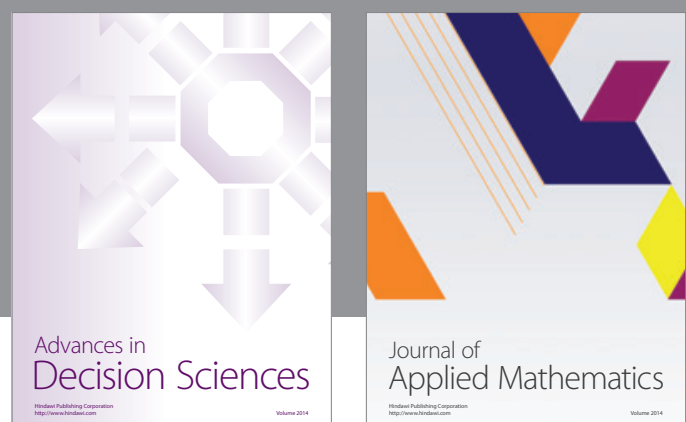

Journal of

Applied Mathematics
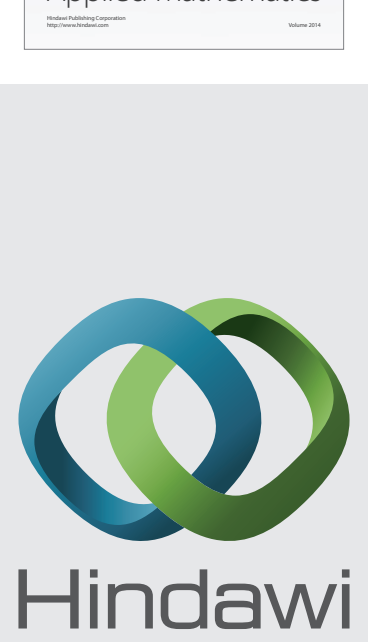

Submit your manuscripts at http://www.hindawi.com
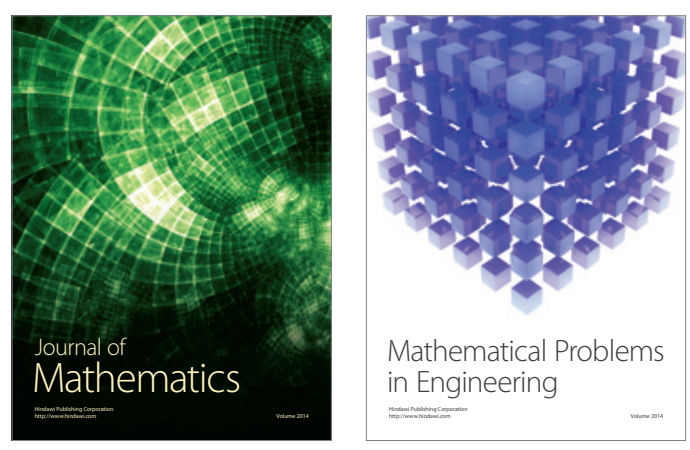

Mathematical Problems in Engineering
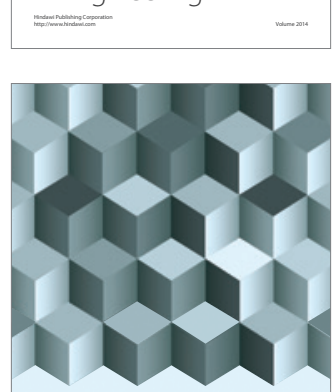

Journal of

Function Spaces
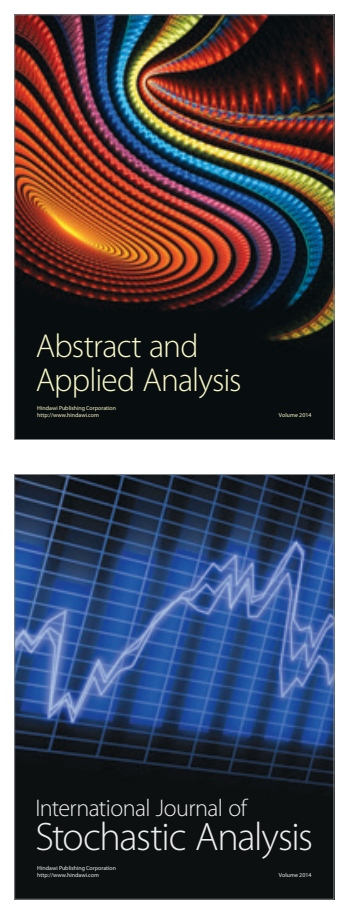

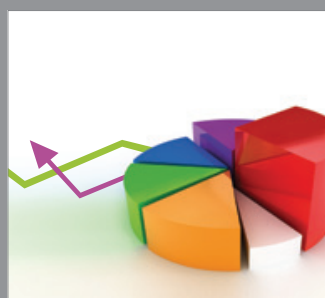

ournal of

Probability and Statistics

Promensencen
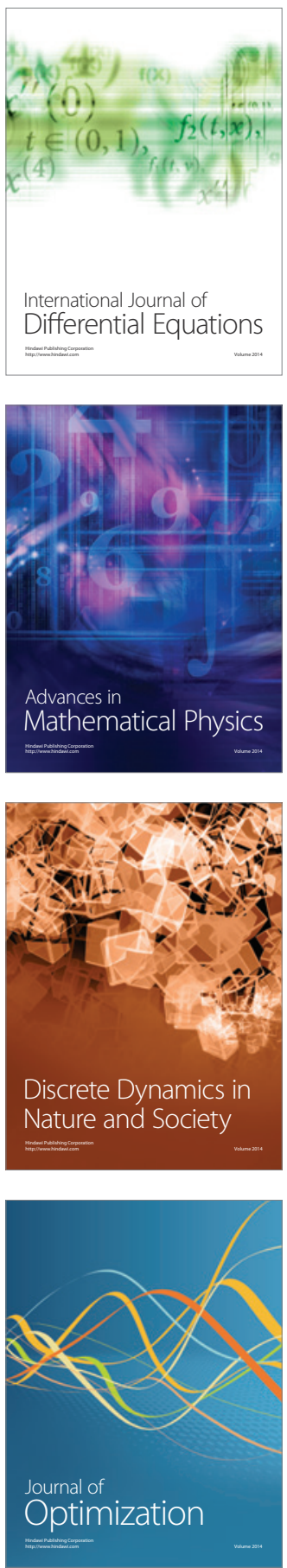